\title{
Numerical Study of the Dynamic Response of Inflatable Offshore Fender Barrier Structures using the Coupled Eulerian-Lagrangian Discretization Technique
}

\author{
Aaron Aboshio $^{+}$and Jianqiao Ye ,* $^{\wedge *}$ \\ ${ }^{+}$Civil Engineering Department, Bayero University, Kano-Nigeria. \\ ${ }^{\wedge}$ Department of Engineering, Lancaster University, Lancaster, LA1 4YR \\ United Kingdom
}

\begin{abstract}
Inflatable Offshore Fender Barrier Structures (IOFBS) are anti-terrorist security structures that function primarily to either stop terror bound vessels from reaching valuable offshore structures, incapacitate its crew or delay the vessel's progress until secondary security measures can be put in place. In this study, an advanced and efficient modelling technique for impact simulation of the structure and similar multi-physics systems is presented. Numerical implementation of this modelling technique, using Abaqus finite element code was described and used in the impact simulation of the inflatable structure based on its current design as well as an alternative design of the structure following impact of a typical vessel hull were carried out and results from the two designs provisions were compared. From the results, recommendations for improvement of the current design are also reported. This is desirable in ensuring high reliability of the structure in meeting its design objectives.
\end{abstract}

Keywords: Inflatable structures, Coupled Eulerian-Lagrangian, Finite element method, Impact Loading, Offshore barrier, Dynamic analysis, Fluid-Structure Interaction

\subsection{Introduction}

The need to protect offshore facilities from deliberate terror attack is of increasing priority especially after the USS Cole (DDG 67) terror incident [2] and more recently the Exxon mobil offshore facility terror attack [3].

One of the viable and effective anti-terrorist barrier structure used around the globe is the inflatable offshore barrier structure developed by Dunlop Holdings ltd. Manchester, UK. The barrier is made majorly of neoprene-nylon fibre reinforced composite of anisotropic mechanical behaviour $[1,4]$. The composite is constructed as units of inflatable tubular structures with operating inflation pressure of $7 \mathrm{kPa}$ (see Figure 1). Each end of the barrier is furnished with mild steel fitting for connections with adjacent segments (units) of the barrier and a reinforcing wire rope is internally connected to the two ends of the steel plate fitting.

- Correspondence author; j.ye2@lancaster.ac.uk 
Current design of the structure stipulates the dimensions of each segment (Figure 1) as single-chambered tubular structure of $2.4 \mathrm{~m}$ diameter, tapering at its ends and with an overall length of $25 \mathrm{~m}$ following recommendation from physical test of the former $1.8 \mathrm{~m}$ diameter structure [2].

IOFBS are traditionally installed on sea site with support conditions provided by mooring systems anchored at the sea bed at predetermined intervals based on environmental weather conditions of the site. Study of the current design under impact [21], following validation of numerical results of the physical tests [5,22] has identified short fall in the level of performance of the enclosed fluid after impact-a key parameter in ensuring desirable dynamic response of the structure in maintaining both its shape, stiffness and strength. Among other important parameters obtained from the study, the results indicated that only $17 \%$ increase of initial inflation pressure in the structure was obtained as the maximum initial surge in pressure following impact of a typical vessel whose physical characteristics are defined in the body of this work.

It is important to note that while low surge in pressure after impact is desirable in some crash structures e.g inflatable bridge rail, bumpers and general adaptive structures [6,7]; it is here conflicting with the overall objective of IOFB structures where high surge of pressure in the enclosed fluid is to be maximised-a condition which basically ensures increased instability of the impactor and potential for high reactive forces and deceleration of the impactor. Thus, to ensure high local surge of pressure (i.e at the point of impact) in the enclosed fluid within allowable limits of the physical properties (strength and stiffness) of the material used for the structure, the current design of the inflatable offshore fender barrier structure is here modified to meet this important property among others.

Although fluid-structure interaction problems can be modelled and solved using the Arbitrary Lagrangian-Eulerian (ALE) formulation [8-10], use here is made of the Coupled Eulerian-Lagrangian (CEL) formulation [11-13] since high structural and fluid displacements and distortions are expected and for which, in numerical analysis, the ALE mesh could lead to unrealistic results and could even crash ongoing simulation[13]

The fluid motion in CEL is defined using the traditional Eulerian description/mesh where the numerical grid is fixed in space while the physical material (water) flows through the grid. The inflatable structure however, is defined using the traditional Lagrangian description of motion and discretized using the Lagrange method where in this case, the numerical grids moves and deforms with the material.

Giving the multi-physics and complex nature of the system under study, an advanced modelling technique for numerical analysis of this and similar FSI problems is presented in this paper. Here, mathematical models employed for the description of fluid behaviour were specifically chosen to ensure computational efficiency for the analysis of large Fluid Structure Interaction (FSI) models. The 
method utilises a single frame, simultaneous solution procedure as well as a unique fluid-structure coupling technique in Abaqus finite element code. This is against the partition method for numerical analysis of FSI problems where the fluid and structure domains are analysed separately and solution variables are iteratively passed from one field to the other at each time or load step. For systems with strong coupling between the enclosed fluid and structure as the one being considered here, very large amount of iteration is required and hence more computationally expensive than the simultaneous method [14].

This paper extends the work reported by Aboshio et al. [15] by explaining the state of the art being addressed and the governing equations for which the numerical results were obtained. Detail description of the FSI model used and numerical implementation of the model for analysis and simulation of the barrier under impact is also presented.

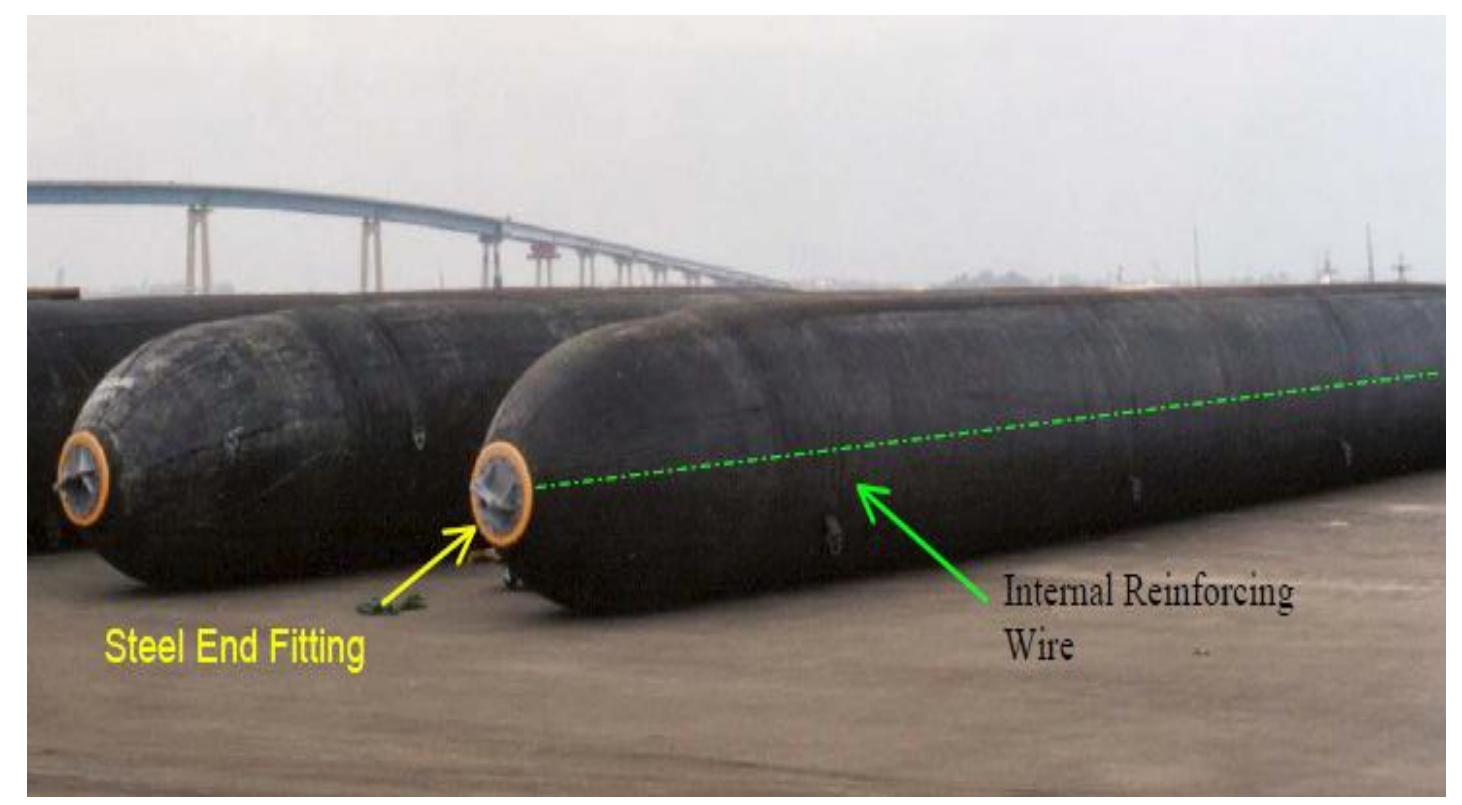

Figure1: Typical segment (unit) of Inflatable Offshore Fender Barrier Structures [2]

\subsection{Governing Equations}

In the Coupled Eulerian-Lagrangian formulation both the Eulerian and the Langrangian equations retain their classical definitions. Material time derivative $\left(\frac{D}{D t}\right)$ is used for solids or structures while spatial time derivative is used for fluid. These are related by:

$\frac{D \phi}{D t}=\frac{\partial \phi}{\partial t}+\mathbf{v} \cdot \nabla \phi$

- Correspondence author; j.ye2@lancaster.ac.uk 
where $\phi$ is an arbitrary solution variable, $\mathbf{v}$ is the material velocity, $\frac{D \phi}{D t}$ and $\frac{\partial \phi}{\partial t}$ are the material and spatial time derivatives of $\phi$ respectively.

Thus, the Lagrangian mass, momentum and energy conservation equations are respectively given by:

$\frac{D \rho}{D t}+\rho \nabla \cdot \mathbf{v}=0$

$\rho \frac{D \mathbf{v}}{D t}=\nabla \cdot \sigma+\mathbf{f}$

$\frac{D E}{D t}=\nabla \cdot(\sigma \cdot \mathbf{v})+\mathbf{f}$

where $\mathrm{E}$ is the sum of kinetic energy and internal energy $(e)$, given as,

$E=1 / 2 \rho \mathbf{v} \cdot \mathbf{v}+e$

for which Equation (4) can alternatively be written as:

$\frac{D e}{D t}=\sigma: \mathbf{D}$

where $\mathbf{D}$ is the velocity strain tensor, $\rho$ is the density and $\mathbf{f}$ is the body force [17].

The Eulerian mass, momentum and energy conservation equations on the other hand read respectively as:

$\frac{\partial \rho}{\partial t}+\nabla \cdot(\rho \mathbf{v})=0$

$\rho \frac{\partial \mathbf{v}}{\partial t}+\nabla \cdot(\rho \mathbf{v} \otimes \mathbf{v})=\nabla \cdot \sigma+\mathbf{f}$

$\frac{\partial e}{\partial t}+\nabla \cdot(e \mathbf{v})=\nabla \cdot(\sigma \cdot \mathbf{v})+\mathbf{f} \cdot \mathbf{v}=\sigma: \mathbf{D}$

The governing equation thus has the general conservation form [17-18]

$\frac{\partial \phi}{\partial t}+\nabla \Phi(\phi, \mathbf{v}, \mathbf{x}, t)=\mathbf{S}$

Where $\phi$ is the arbitrary solution variable, $\mathbf{x}$ is the spatial coordinate, $\Phi$ is the flux function, $\mathbf{S}$ is the source term and $t$ is time. Using operator splitting [17], Equation (9) divides into Equations (10) and (11) which respectively are referred as the Lagrangian step and the Eulerian step which accounts for the transport of the material (water) between the Eulerian elements.

$$
\frac{\partial \phi}{\partial t}=\mathbf{S}
$$




$$
\frac{\partial \phi}{\partial t}+\nabla \cdot \Phi(\phi, \mathbf{v}, \mathbf{x}, t)=0
$$

\section{$2.1 \quad$ Fluid-Structure Coupling}

For the analysis of the structure under study in this paper, two coupling scenarios were considered. First is the coupling/interaction between enclosed fluid and the structure and secondly the coupling/interaction between the inflatable (enclosed fluid plus structure) and water on which the structure floats.

Figure 2 presents the coupling scenario where the Lagrangian domain $\Gamma$, can be solved in the Lagrangian step and Eulerian domain $\mathrm{K}$, solved during the Eulerian step. Here the enclosed fluid (air) behaviour is described using the ideal gas law for computational efficiency as when compared to description of the fluid behaviour using the Euler or Navier stokes equations [5,7].

Pressure from the enclosed fluid at the fluid-structure interface can be analytically computed using the relation in Equation (12).

$\overline{\mathbf{P}}=\rho R \bar{\theta}$

or

$\rho(\rho, \theta)=\frac{\mathbf{P}+P_{A}}{R\left(\theta-\theta_{Z}\right)}$

where $\overline{\mathbf{P}}$ is the absolute pressure, $\mathbf{P}$ is the gauge pressure, $p_{A}$ is the ambient pressure, $R$ is the gas constant, $\rho$ is the fluid density, $\theta$ and $\theta_{Z}$ are the current and absolute zero temperatures respectively.

The gas constant, $R$, can also be determined from the universal gas constant, $\bar{R}$, and the molecular weight, $M_{w}$, given by

$$
R=\frac{\bar{R}}{M_{w}}
$$

Since, in this problem, no heat is added or removed from the system except for the initial predetermined field temperature in the cavity, the fluid temperature following a dynamic event can be determined using the energy balance equation, given by:

$\Delta E=\Delta Q-\Delta W$

where $\Delta E$ is the internal energy, $\Delta Q$ is the energy transferred into the system and $\Delta W$ is the work done by the enclosed air.

- Correspondence author; j.ye2@lancaster.ac.uk 


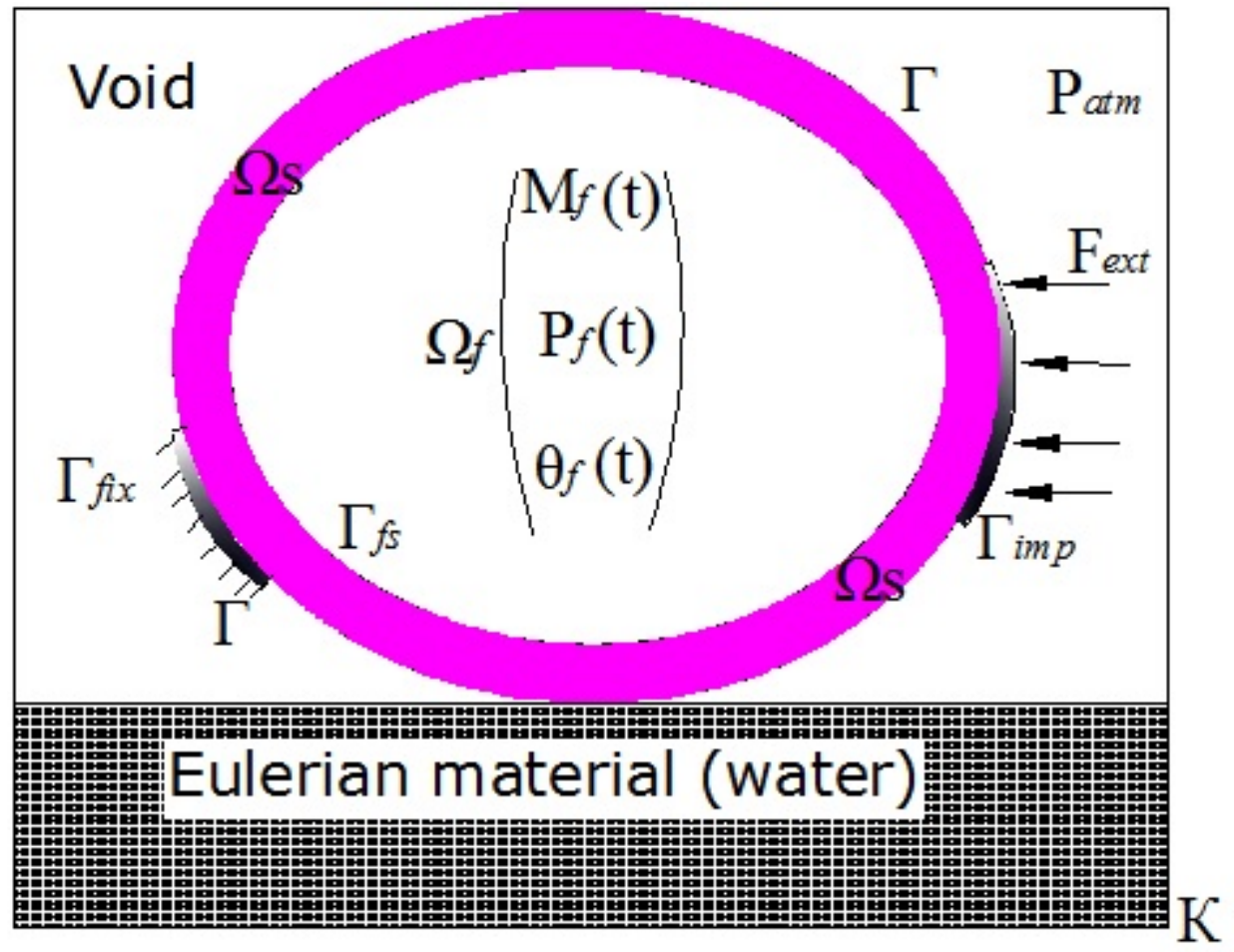

Figure 2: Fluid Structure Interaction Model (where $m_{f}=$ mass of fluid, $p_{f}=$ gauge pressure of fluid and $\theta$ is the temperature of the fluid)

In Figure 2, let the Lagrangian domain, $\Gamma$, occupied by solid be $\Omega_{s}$, the domain occupied by fluid be $\Omega_{f}$ and the direct fluid-structure interaction, (i.e where the structure interacts with the fluid-the internal part of the solid body) be denoted $\Gamma_{f s}=\Omega_{s} \cap \Omega_{f}$. $\Gamma_{f i x}$ is the external boundary where displacement conditions are imposed and $\Gamma_{i m p}$ is where stress boundary conditions are specified.

Thus, the boundary conditions of the solid part can be written as:

$$
\begin{array}{lll}
\mathbf{v}_{s}=0 & \text { on } & \Gamma_{f i x} \\
\sigma_{s} \mathbf{n}=F_{\text {ext }} & \text { on } & \Gamma_{\text {imp }}
\end{array}
$$

and the boundary conditions of the fluid are defined as:

$$
\mathbf{v}_{f}=0 \quad \text { on } \quad \Gamma_{f i x}
$$

The coupling conditions defined at the $\Gamma_{f s}$ boundary are :

- Correspondence author; j.ye2@lancaster.ac.uk 
$\sigma_{s} \mathbf{n}=\sigma_{f} \mathbf{n}+F_{e x t}$

$\mathbf{v}_{f}=\mathbf{v}_{s}$

where $\sigma_{f} \mathbf{n}=-\bar{p} \mathbf{n} ; \bar{p}$ is the average gauge pressure in the structure; $\mathbf{n}$ is the unit normal vector; $F_{\text {ext }}$ is the impact force; and $\sigma_{s}, \sigma_{f}, v_{s}$ and $v_{f}$ are the solid and fluid Cauchy stresses and velocities, respectively.

Giving the strong coupling between the enclosed fluid and the structure, a monolithic or simultaneous approach using the Lagrange equations was used for numerical analysis of the inflatable structure.

The Lagrangian momentum equation coupling the structure and the enclosed fluid can be written in finite element form as:

$\mathbf{M} \ddot{\mathbf{U}}+\mathbf{C} \dot{\mathbf{U}}+\mathbf{K U}+\mathbf{F}^{\mathrm{int}}(\overline{\mathbf{P}}, \mathbf{U})=\mathbf{F}^{\text {ext }}$

subject to the following initial conditions

$\mathbf{U}(0)=\mathbf{U}^{0}, \dot{\mathbf{U}}(0)=\mathbf{V}^{0}$

Where $\mathbf{M}, \mathbf{C}$ and $\mathbf{K}$ are the mass, viscous damping and stiffness matrices of the solid structure, respectively; $\mathbf{U}$ is the displacement vector; $\mathbf{F}^{\text {int }}$ is the force from the enclosed fluid as a function of the fluid pressure and displacement at the fluid structure interface and $\mathbf{F}^{\mathrm{ext}}$ is the external loading on the structure. $\overline{\mathbf{P}}=\mathbf{P}+P_{A}$, where $\overline{\mathbf{P}}$ is the average absolute pressure, $P_{A}$ is the ambient pressure and $\mathbf{P}=\left\{P_{1}, P_{2}, P_{3}, \ldots P_{n}\right\}$ being the gauge pressures in the individual chambers of the proposed multi chamber inflatable structure.

The second coupling for the problem under consideration involves the interaction between the inflatable structure described above and the sea water on which the structure floats. To account for this scenario the Coupled Eulerian-Lagrangian formulation is employed where the inflatable structure was analysed based on classical Lagrange equation of motion as mentioned earlier and the fluid (water), was analysed based on the classical Eulerian-Lagrangian formulation.

The sea water behaviour was described using the Equation of State (EOS) presented in Equation (19). This was assigned to the Eulerian mesh as fraction of the Eulerian domain while allowing also for void region within the mesh/model as shown in Figure 2.

The sea water-inflatable structure coupling was achieved via contact interaction algorithm between the two bodies. Numerical technique for achieving this involves

- Correspondence author; j.ye2@lancaster.ac.uk 
embedding the Lagrangian structure in the void region of the Eulerian mesh. Contact between the Lagrange structure and the Eulerian material interface can be enforced using the 'penalty' method as described in $[5,13,19]$. From this, the structure imposes displacement or velocity boundary condition on the fluid (water), while the fluid imposes traction boundary condition on the structure.

$$
P=\frac{\rho_{0} c_{0}^{2} \eta}{(1-s \eta)^{2}}\left(1-\frac{\Gamma_{0} \eta}{2}\right)+\Gamma_{0} \rho_{0} E_{m}
$$

where, $\eta=1-\frac{\rho_{0}}{\rho}$ is the nominal volumetric compressive strain; $\Gamma=\Gamma_{0} \frac{\rho_{0}}{\rho}$ is the Gruneisen ratio with $\Gamma_{0}$ being the material constant; $E_{m}$ is the energy per unit mass; $P$ is the pressure; $\rho_{0} c_{0}^{2} \eta$ is equivalent to the elastic bulk modulus at a small nominal strain; $\rho_{0}$ and $\rho$ are the reference and current densities, respectively.

The water wave speed $\left(c_{0}\right)$, density and shear viscosity considered in this study are, respectively, $1483 \mathrm{~m} / \mathrm{s}, 1000 \mathrm{~kg} / \mathrm{m}^{3}$ and $0.001 \mathrm{~N} . \mathrm{sm}^{-2}$.

\subsection{Numerical model}

The fluid structure interaction model described in Section 2 was effectively, numerically implemented in Abaqus finite element code. The model set-up in Abaqus is very similar to the framework presented in Figure 2.

Here, Eulerian domain was numerically modelled using cube/volume Eulerian elements (EC3D8R). The mesh from this elements were partly filled with water material modelled using Equation (19) and the remaining left with no material assigned (voided) as can be seen in Figure 2. Material assignment in the Eulerian domain was achieved using the Eulerian Volume Fraction (EVF) in the 'predefined fields'. EVF equal to one (1) implies that the Eulerian mesh segment is completely filled with the material, while if EVF is zero, the mesh is however completely voided.

The Inflatable structures however, were modelled using Lagrangian membrane elements (M3D8R), impacting vessel modelled using C3D10M elements and the connector elements (CONN3D2) for shackles and mooring system of the structure as described in Section 1. Material properties of the structure's carcasses and the impacting vessel are presented in the following sub sections. They were modelled and assigned as described in [19] to the structure and vessel accordingly for the impact simulation.

For all numerical analysis of the IOFBS presented in this paper, the barrier units were initially inflated to $7 \mathrm{kPa}$. The pressure was prescribed at the barrier cavity

- Correspondence author; j.ye2@lancaster.ac.uk 
reference node using boundary conditions. This has the effect of automatically adjusting the enclosed fluid volume to fill the barrier units/cavities so as to maintain the prescribed $7 \mathrm{kPa}$ pressure [19]. In the subsequent steps, the pressure boundary condition was suppressed to allow for rise of the enclosed pressure as the structure deforms under the impact loading.

Section 4 presents the structural models and inflation pressure adopted in this study as also described in Section 2.

\subsection{Properties of Components of the Inflatable Structure \\ 3.1.1 Barrier Carcasses}

The barrier shell is made of M006 barrier composites material made from neoprene polymer matrix and woven nylon (nylon 6.6) fibres [20]. The structure is constructed with the composite reinforcement oriented in the warp direction corresponding to the longitudinal length of the barrier structure and the cap ends material oriented to the weft direction which corresponds to the circumferential direction of the structure.

Experimental (uniaxial) test carried out on the composites indicates that the composites demonstrate anisotropic mechanical properties with dissimilar results obtained for specimens oriented in the warp and weft directions [1,4,5]. Table 1 presents properties of the composite material considered in this study.

\begin{tabular}{|l|l|l|l|l|}
\hline Parameters & Warp & Weft \\
\hline Ultimate Strength (MPa) & 61.51 & 47.72 \\
\hline Ultimate Strain & 0.29 & 0.37 & \multicolumn{2}{|l|}{} \\
\hline $\begin{array}{l}\text { Bilinear Modulus (MPa) } \\
\text { (Primary and secondary } \\
\text { modulus respectively) }\end{array}$ & 144.66 & 295.47 & 50.92 & 150.96 \\
\hline Density (kg/m ${ }^{3}$ ) & 1287 & \multicolumn{3}{|l|}{} \\
\hline Nominal Thickness (mm) & 4.00 & \multicolumn{4}{|l|}{} \\
\hline
\end{tabular}

Table 1: Material uni-axial properties of neoprene-nylon fibre reinforced composites

\subsubsection{End plate fittings and internal reinforcement}

The end plate fittings were made of mild steel plates that are 0.6 metres in diameter and 0.1 metres thick. The modulus and density of the material are, respectively, 205 $\mathrm{GPa}$ and $7850 \mathrm{~kg} / \mathrm{m}^{3}$. The internal reinforcement were made of $24 \mathrm{~mm}$ diameter wires of $991.94 \mathrm{MPa}$ ultimate strength and modulus of $63.74 \mathrm{MPa}$.

\subsection{Vessels/Boats}

- Correspondence author; j.ye2@lancaster.ac.uk 
A High Speed Manoeuvring Surface Target (HSMST) vessel with characteristic parameters presented in Table 2 was use for this study. This boat represents medium surface threats that are taken to be readily available to terrorist and paramilitary groups [2]. Geometric shape of the vessel front edge was kept blunt to avoid local element damage or excessive distortions during contact with the barrier. The composite material is known to likely tear following vessel propeller cut, sharp egde thrust or as a results of gun shots [2,5].

\begin{tabular}{|l|l|}
\hline Vessel Characteristics & HSMST Boat \\
\hline Length $(\mathrm{m})$ & 7.239 \\
\hline Draft $(\mathrm{m})$ & 0.814 \\
\hline Beam $(\mathrm{m})$ & 2.743 \\
\hline Dead weight $(\mathrm{kg})$ & 1837 \\
\hline Impact Speed $(\mathrm{m} / \mathrm{s})$ & 13.4 \\
\hline Kinetic Energy Transferred (MJ) & $0.16 \mathrm{MJ}$ \\
\hline
\end{tabular}

Table 2: HSMST vessel's physical characteristics

\subsection{Geometric Model}

Figure 3 shows units of the geometric model developed in Abaqus CAE based on current design of the inflatable structure and a modified unit with five segments partitioned by internal walls as shown in the figure.

Structural models developed from these in this study consist of three units of the barrier shackled together at the units ends, moored and anchored as shown in Figure 4(A) and 4(B) for the current and modified designs respectively.

A hybrid design, consisting of all the provisions in the above designs, was also considered in this study. 


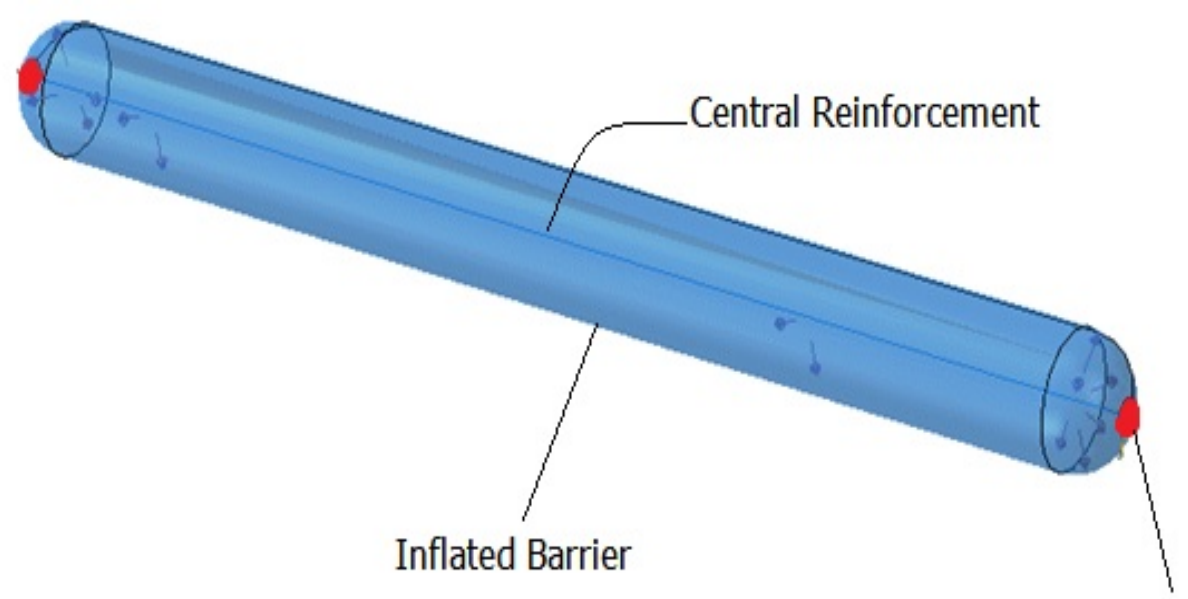

Barrier Endplate

A
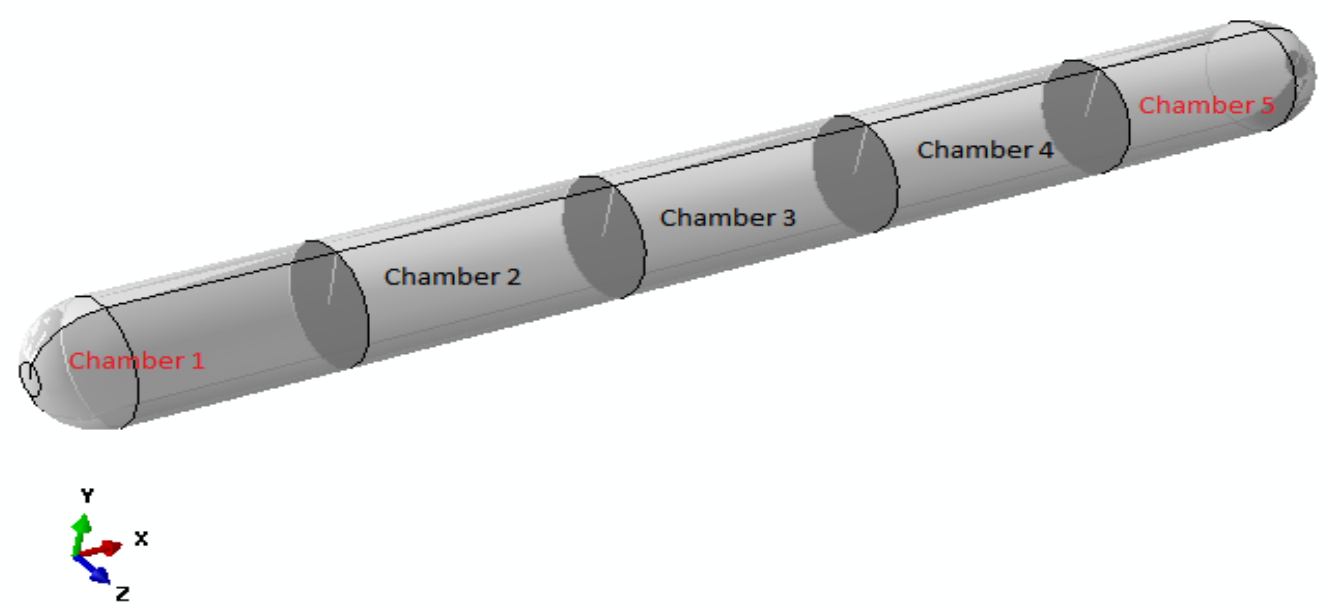

B

Figure 3: Geometric units of the inflatable barrier based on current (A) and modified (B) design provisions

- Correspondence author; j.ye2@lancaster.ac.uk 


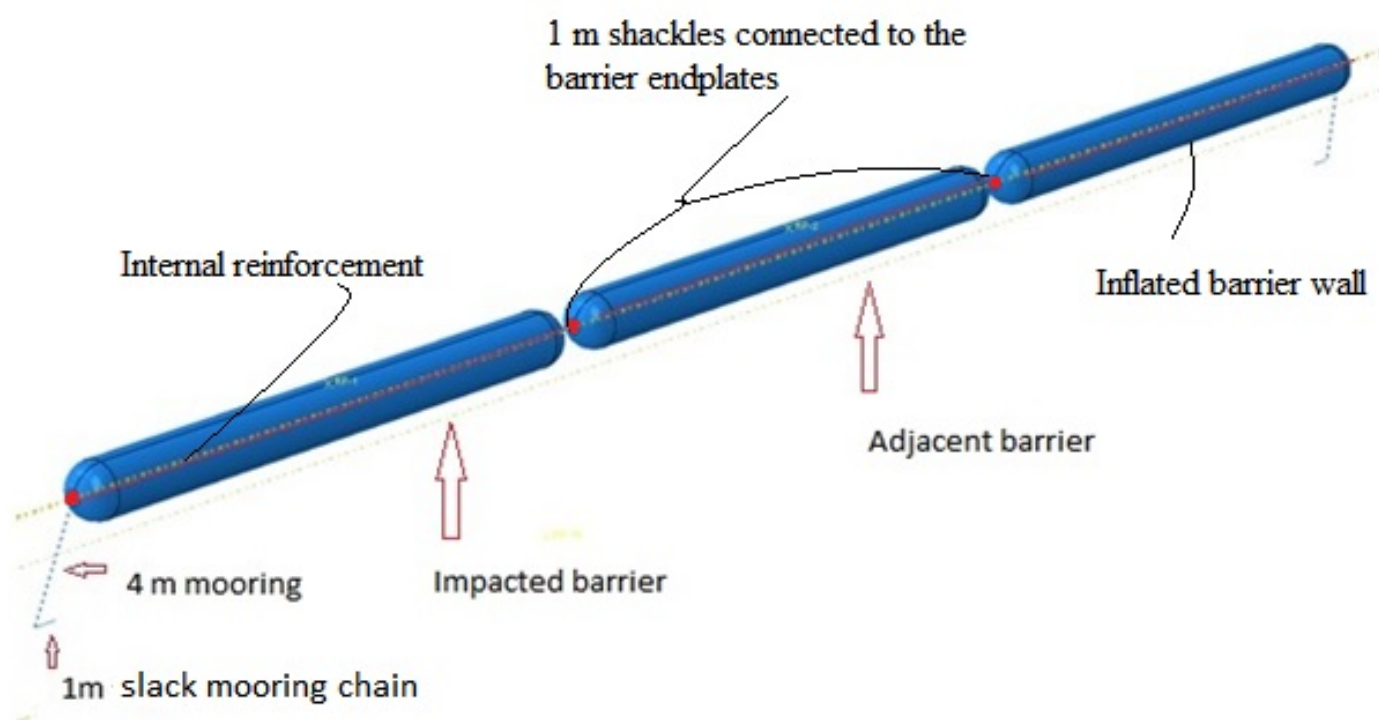

A

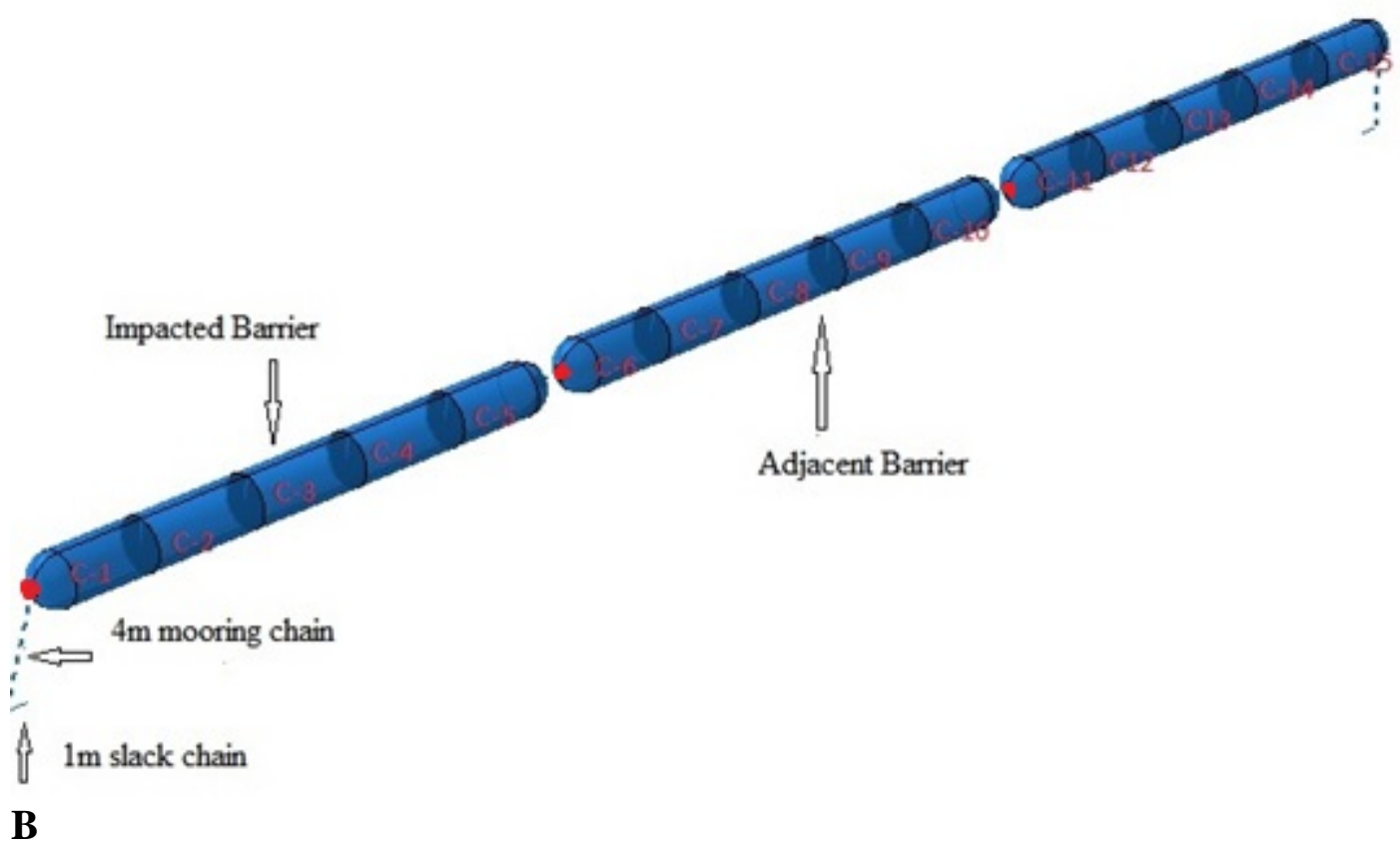

Figure 4: Geometric model of the inflatable offshore fender barrires based on current (A) and modified (B) units.

- Correspondence author; j.ye2@lancaster.ac.uk 


\subsection{Simulation}

Abaqus explicit using the Coupled Eulerian-Lagrangian formulation (CEL) was utilised to analyse the stress distributions, deformations, and the general response of the structure under impact loading of the vessel. The Vessel initial impact on the barrier for all analyses carried out was at a time step of $0.24 \mathrm{~s}$ and full impact time step of $0.32 \mathrm{~s}$.

Standard earth acceleration due to gravity of $9.81 \mathrm{~m} / \mathrm{s}^{2}$ was applied to the whole model at the first load step in order to activate self-masses and determine the initial settlement/displacements of the structures under dead weights and buoyancy loadings. The buoyancy loading is for surfaces below the water level which are subjected to hydrostatic pressure.

The vessel with geometric dimension defined in Table 2 was model and constrained as a rigid body moving at a speed of $13.4 \mathrm{~m} / \mathrm{s}$.

The general contact capabilities available in Abaqus was utilised to simulate contact interactions between the various parts of the model. 'Hard' contact with frictionless tangential behaviour was defined for the whole model (plus $0.001 \mathrm{~N} / \mathrm{m} . \mathrm{s}$ shear viscosity in water). In addition a 'master-slave' contact formulation was defined for the vessel-barrier surface interaction using the penalty contact algorithm discussed earlier.

\subsection{Results and Discussion}

Figures 5 and 6 respectively show maximum principal stress distribution on the barrier walls based on the current and the modified design following a full impact of the vessel. The stress level is slightly higher in the modified design than is in the current design, but can be said to be very similar. The maximum stresses of 13.09 $\mathrm{MPa}$ and 13.65 MPa for the current and modified design, respectively, were recorded and are both much lower than the composite material strength shown in Table 2. Stress distribution in the internal partition walls of the modified design is shown in Figure 7. This is well below the stress distribution on the external barrier wall discussed above, which indicates that the partition wall can keep the enclosed fluid within the chambers through the impact period. Initial study leading to the $5 \mathrm{~m}$ partition wall intervals shows that the closer the internal walls the higher the stresses on the walls will be. However, further increase of the partition distance may result in consequential effect of lower pressure surge after impact.

The maximum strains recorded for the two design provisions are shown in Figures 8 and 9 respectively for the current and modified barrier units. Results from the modified design also show slightly higher strains in the barrier wall than they are in the current design. The maximum strains for the current and modified designs are

- Correspondence author; j.ye2@lancaster.ac.uk 
respectively 0.22 and 0.24 and are again less than the ultimate strain of the composite material presented in Table 2.

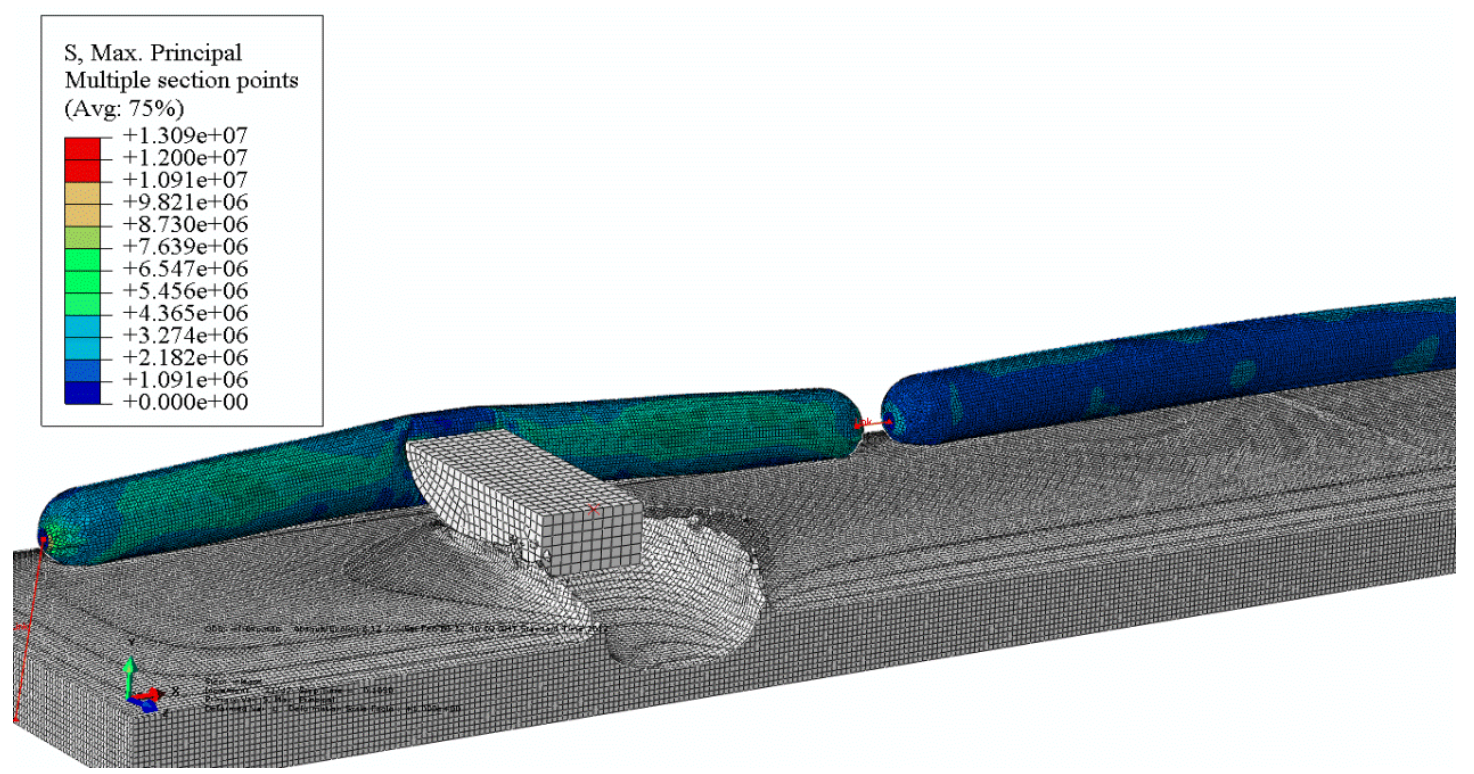

Figure 5: Maximum principal stress distribution at full impact state of current barriers with initial inflation pressure of $7 \mathrm{kPa}$

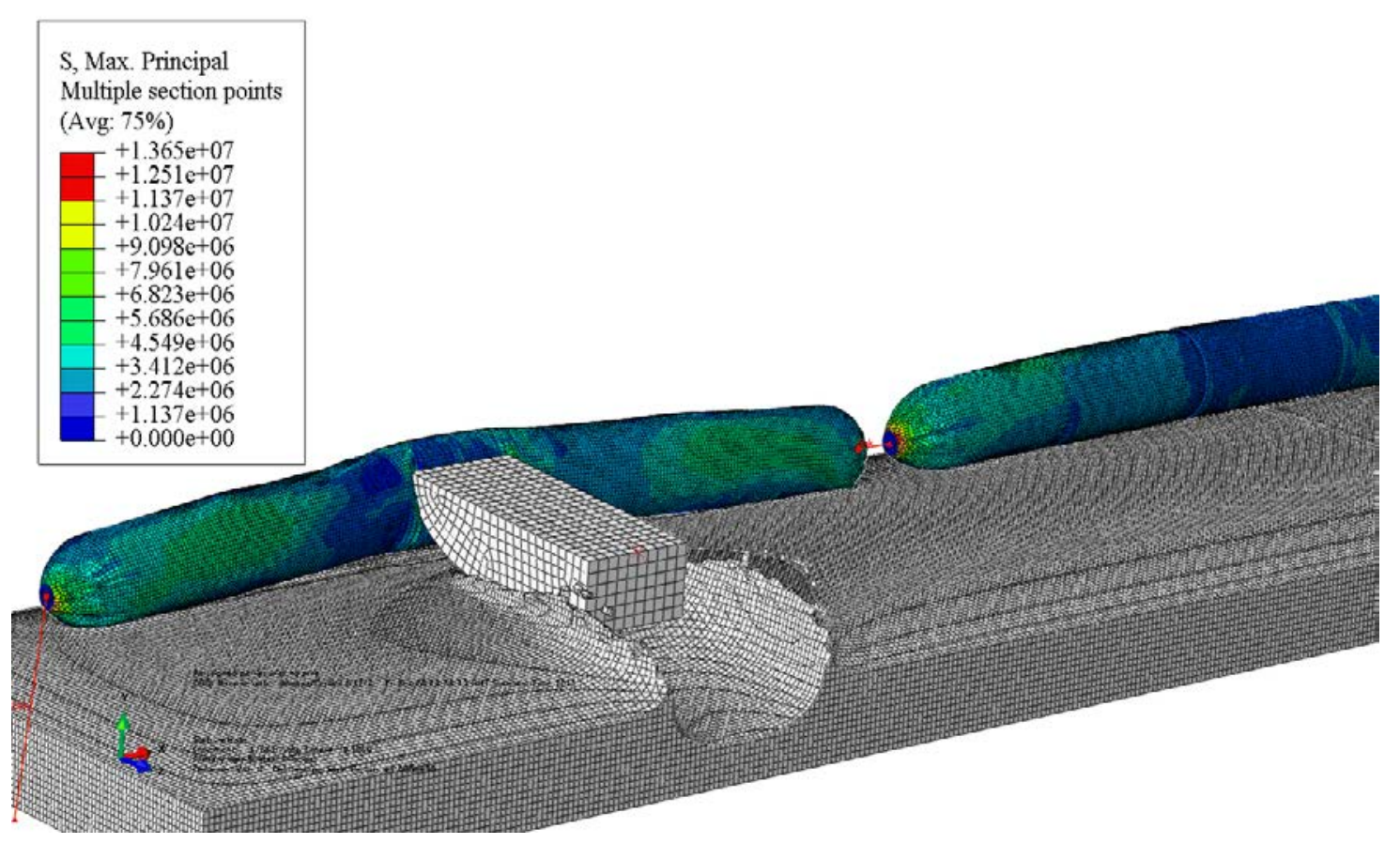

- Correspondence author; j.ye2@lancaster.ac.uk 
Figure 6: Maximum principal stress distribution at the full impact state of modified barrier with initial inflation pressure of $7 \mathrm{kPa}$

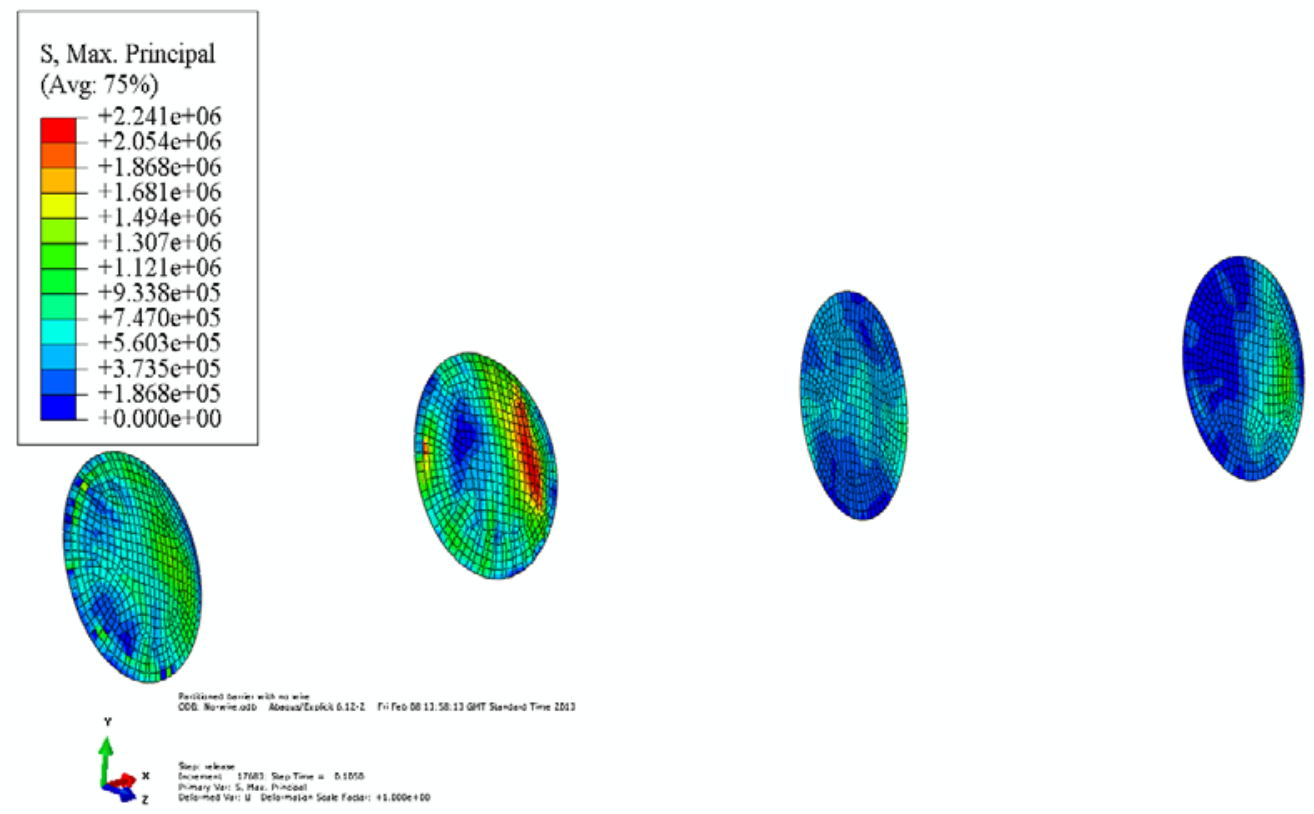

Figure 7: $\quad$ Maximum principal stress distribution at the full impact state of internal partition walls of the modified design with initial inflation pressure of $7 \mathrm{kPa}$

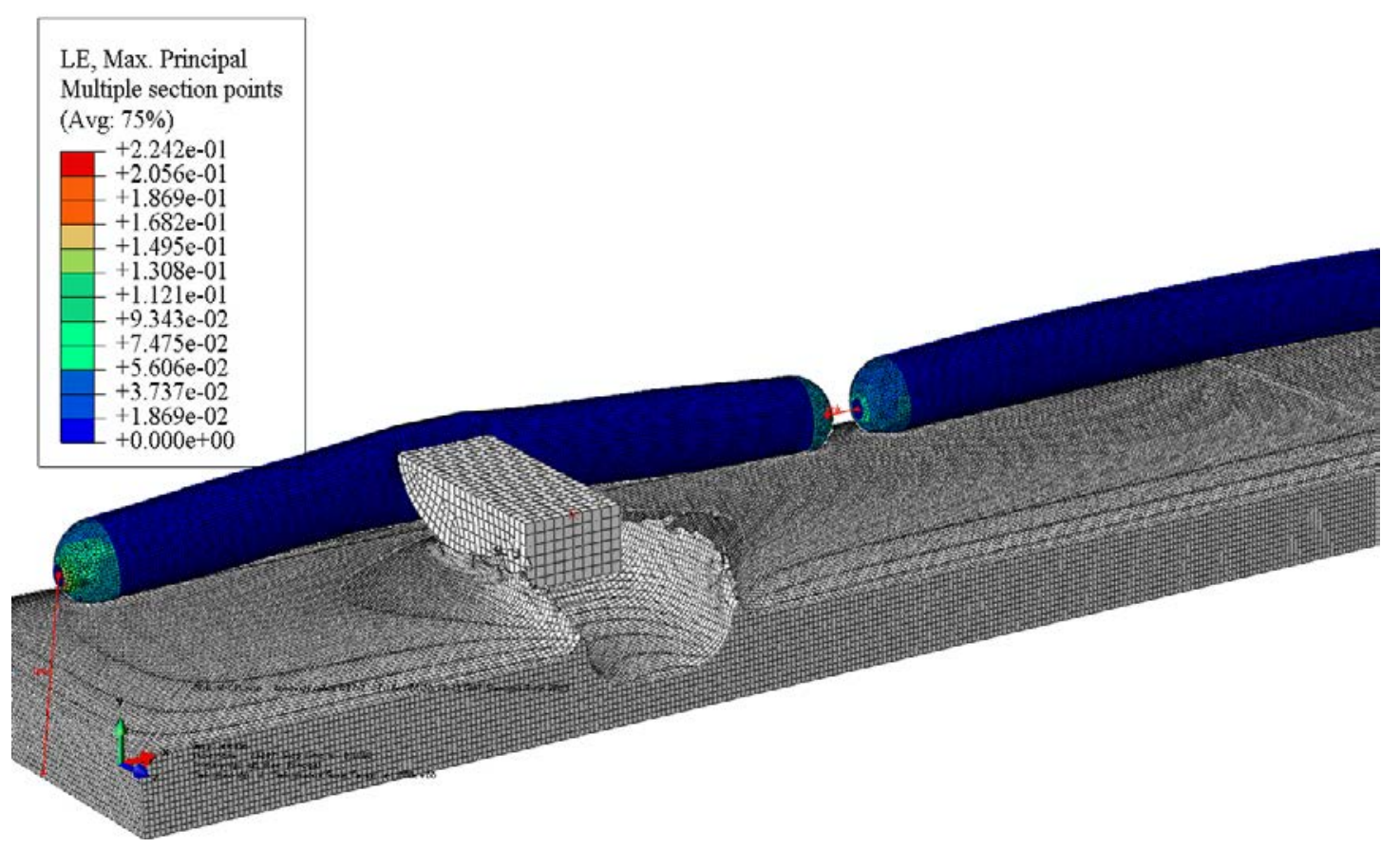

Figure 8: Logarithmic strain distribution at full impact state of current barriers with initial inflation pressure of $7 \mathrm{kPa}$.

- Correspondence author; j.ye2@lancaster.ac.uk 


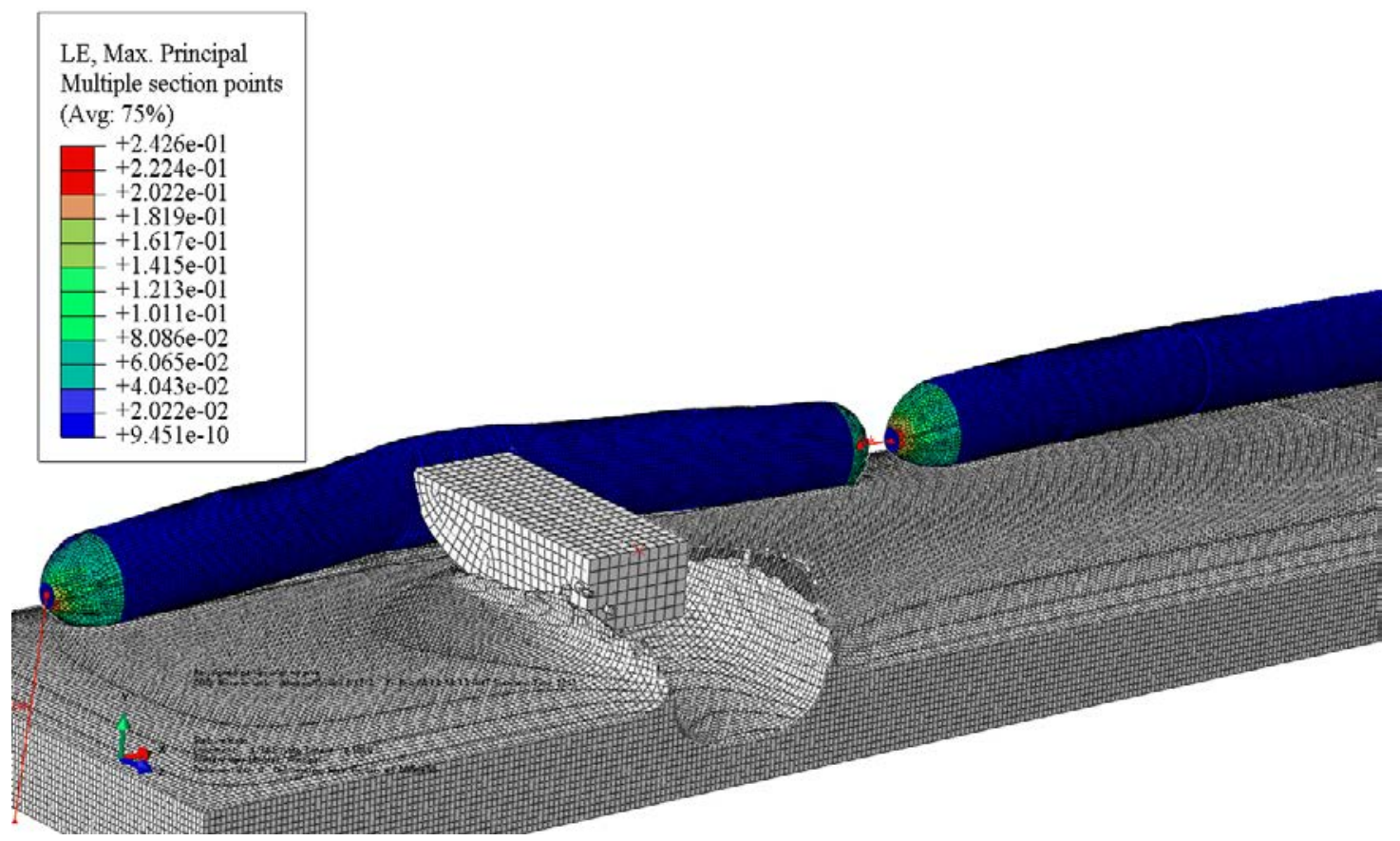

Figure 9: Logarithmic strain distribution at the full impact state of modified barrier with initial inflation pressure of $7 \mathrm{kPa}$

Whilst the comparisons of the stress and strain distributions in the two designs do not show clear gains of the modified structure, the history of pressure of the enclosed air (Figures 10 and 11) following impact of the current and modified designs respectively does. A sharp instantaneous local surge in pressure can be seen in the impacted chamber of the modified design than is in the current design. 105\% increase in pressure within $0.05 \mathrm{~s}$ was recorded for the modified design than the $17 \%$ increase recorded in the current design.

This local instantaneous surge in pressure consequently leads to a higher work done by the enclosed air which functions to dissipate impact kinetic energy of the impacting vessel. Figure 12, shows a significant work done by the enclosed fluid in the modified design as the vessel impact the structure. This high work done by gas can be said to be solely due to the confinement of the enclosed air in the chambers. Relating to Equation (20) below, this observation implies that the work done by the enclosed air affects the stress distribution and load carrying capacity of the barrier.

$$
\Delta E_{k}(t)-\Delta W_{k}=W_{E x t}(t)
$$

where $\Delta E_{k}(t)$ is the change in kinetic energy (KE) of the barrier system; $W_{E x t}(t)$ is the work done by the impacting boat (vessel) and $\Delta W_{k}$ is the work done by the enclosed fluid. 


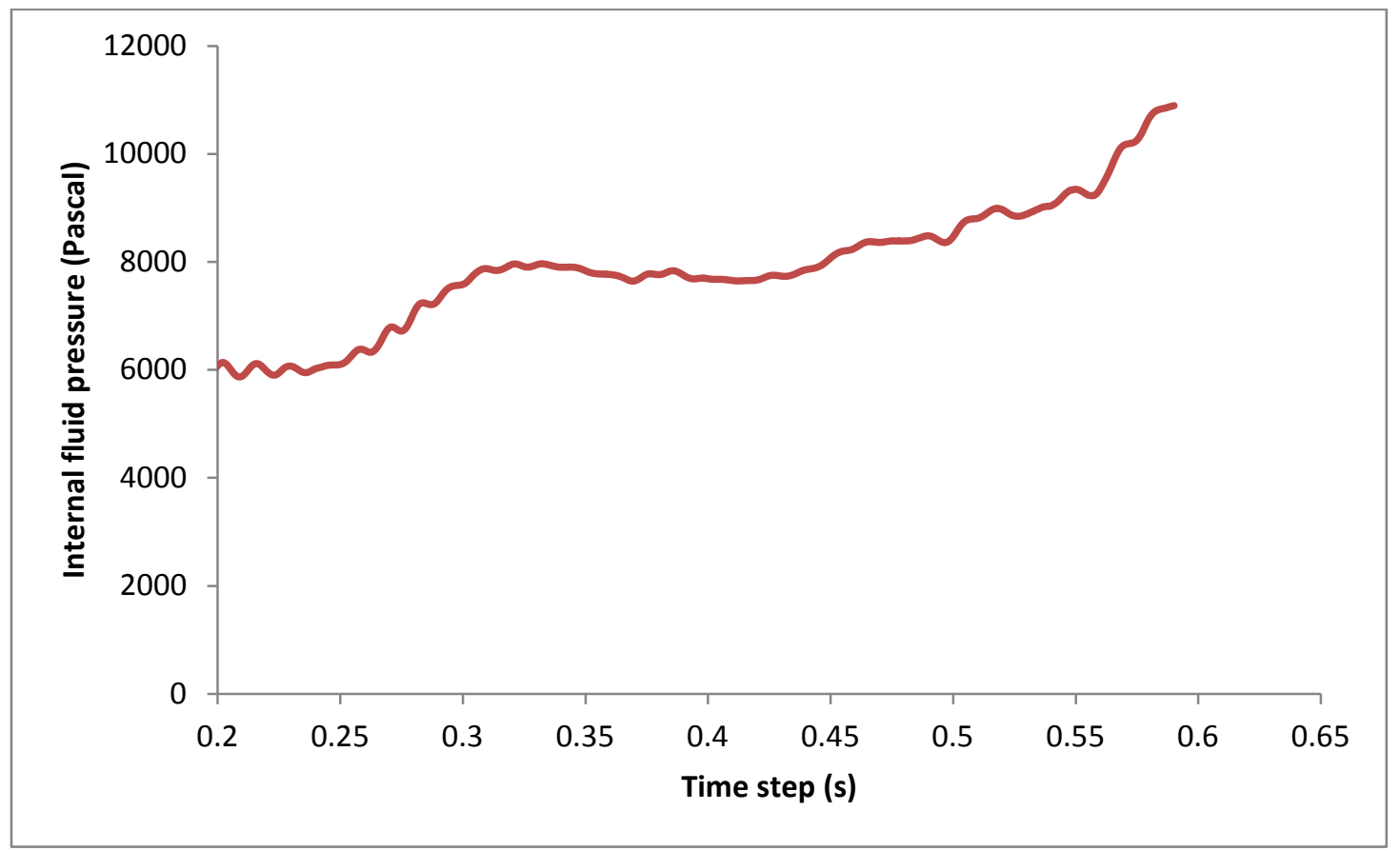

Figure 10: Enclosed fluid pressure variation with time of current barrier inflated to $7 \mathrm{kPa}$

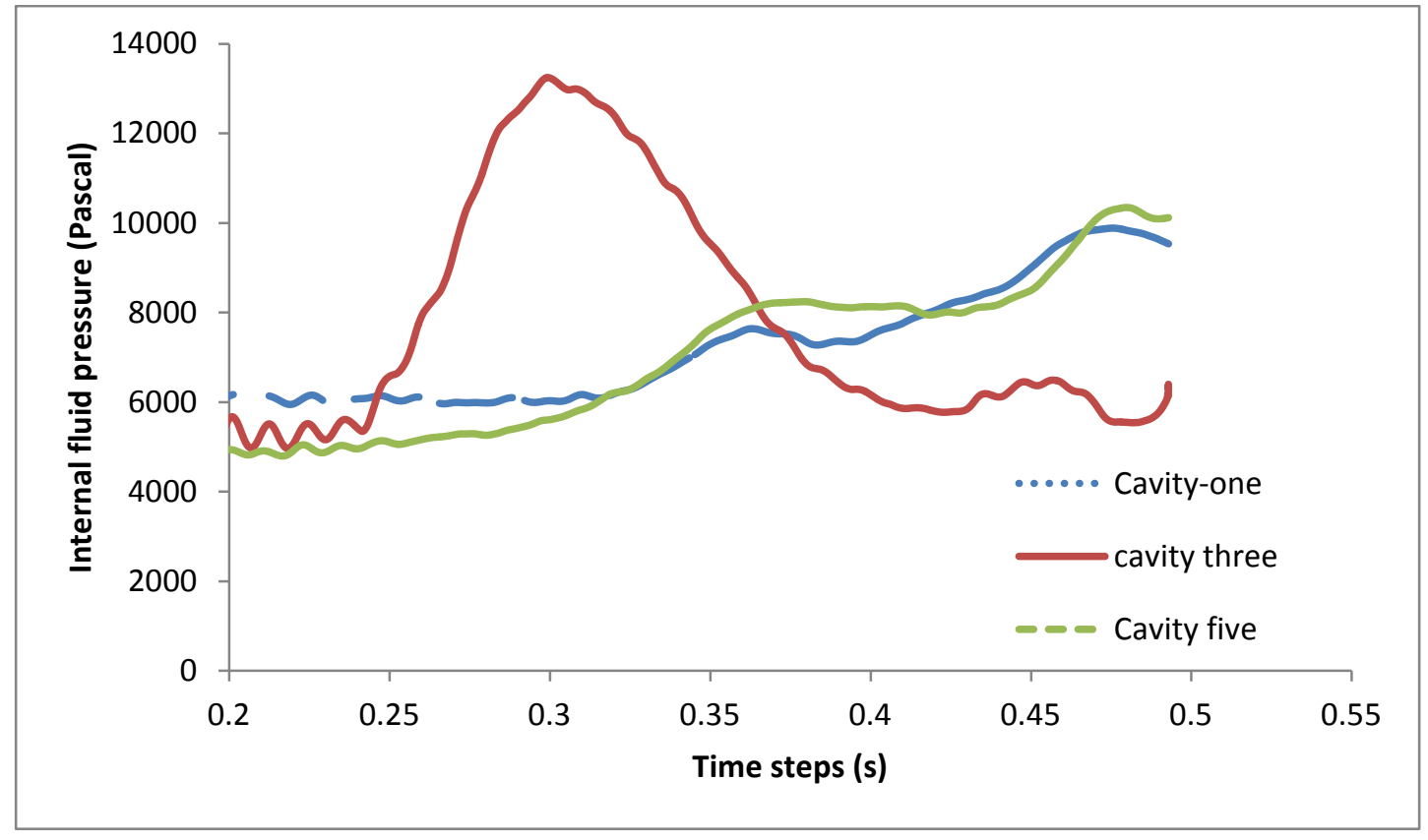

Figure 11: Enclosed fluid pressure variation in chambers 1,3 and 5 with time of modified barrier inflated to $7 \mathrm{kPa}$

- Correspondence author; j.ye2@lancaster.ac.uk 


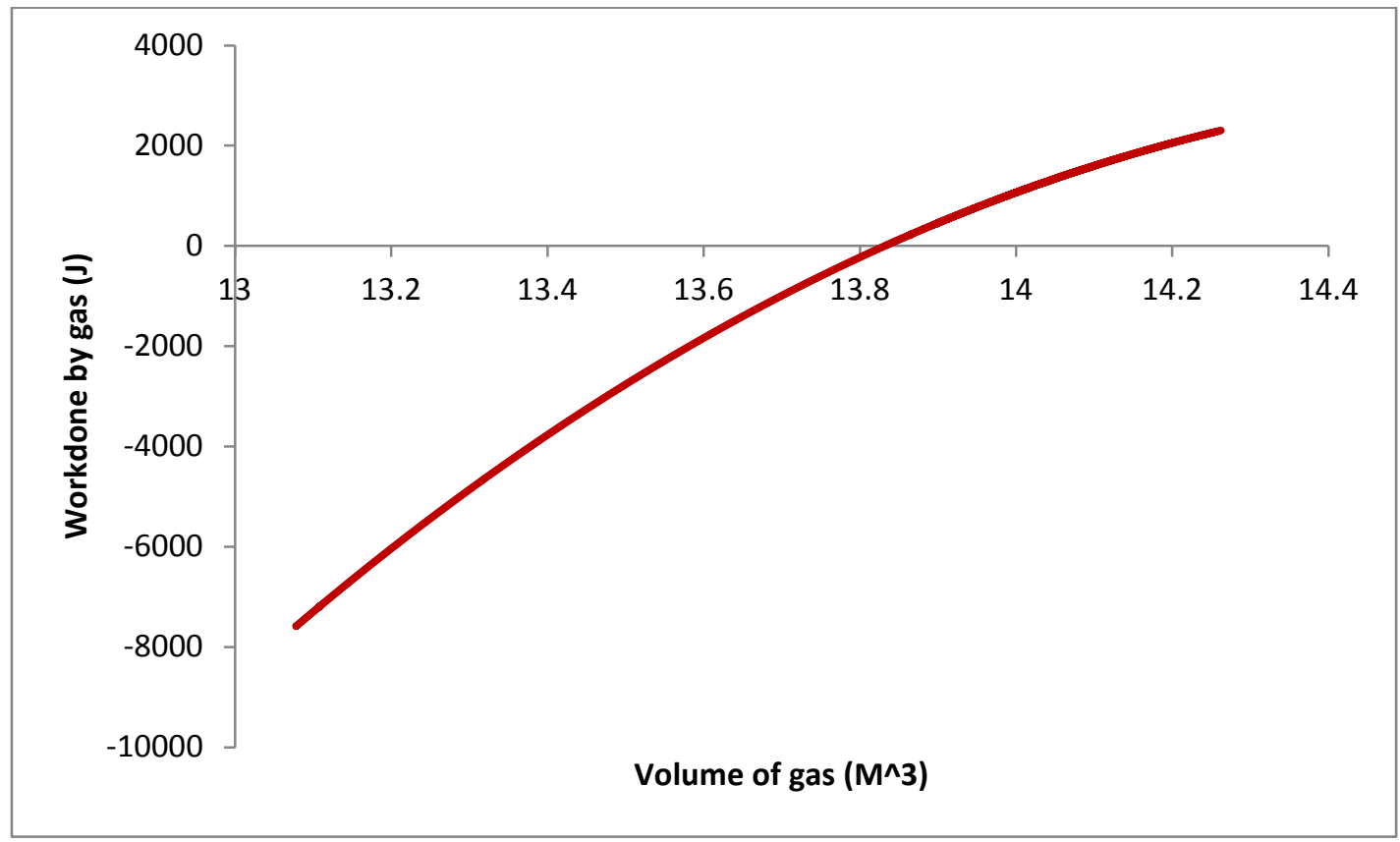

Figure 12: History of work done by enclosed air against volume of air compressed in the impacted chamber of the modified design.

Other plausible gains of the modified design can be seen in the increased level of instability (rotation velocity) of the vessel. Figures 13 and 14 show the rotation velocity for the current and modified designs respectively. From the figures, it can be seen that about 30\% increase in the vessel instability following impact of the modified design was obtained when compared to the current design.

Decelerations of the vessel after impact of the current and modified barrier initially inflated to $7 \mathrm{kPa}$ are presented in Figures 15 and 16 respectively. A maximum deceleration of $367 \mathrm{~m} / \mathrm{s}^{\wedge} 2$ within a period of $0.05 \mathrm{~s}$ of impact was obtained for the modified barrier structure while $132 \mathrm{~m} / \mathrm{s}^{\wedge} 2$ was recorded for the current design. The deceleration values obtained in the modified design surpasses the threshold of $294.3 \mathrm{~m} / \mathrm{s}^{\wedge} 2$ (30g) needed to cause significant distress on vessel's crew [2].

- Correspondence author; j.ye2@lancaster.ac.uk 


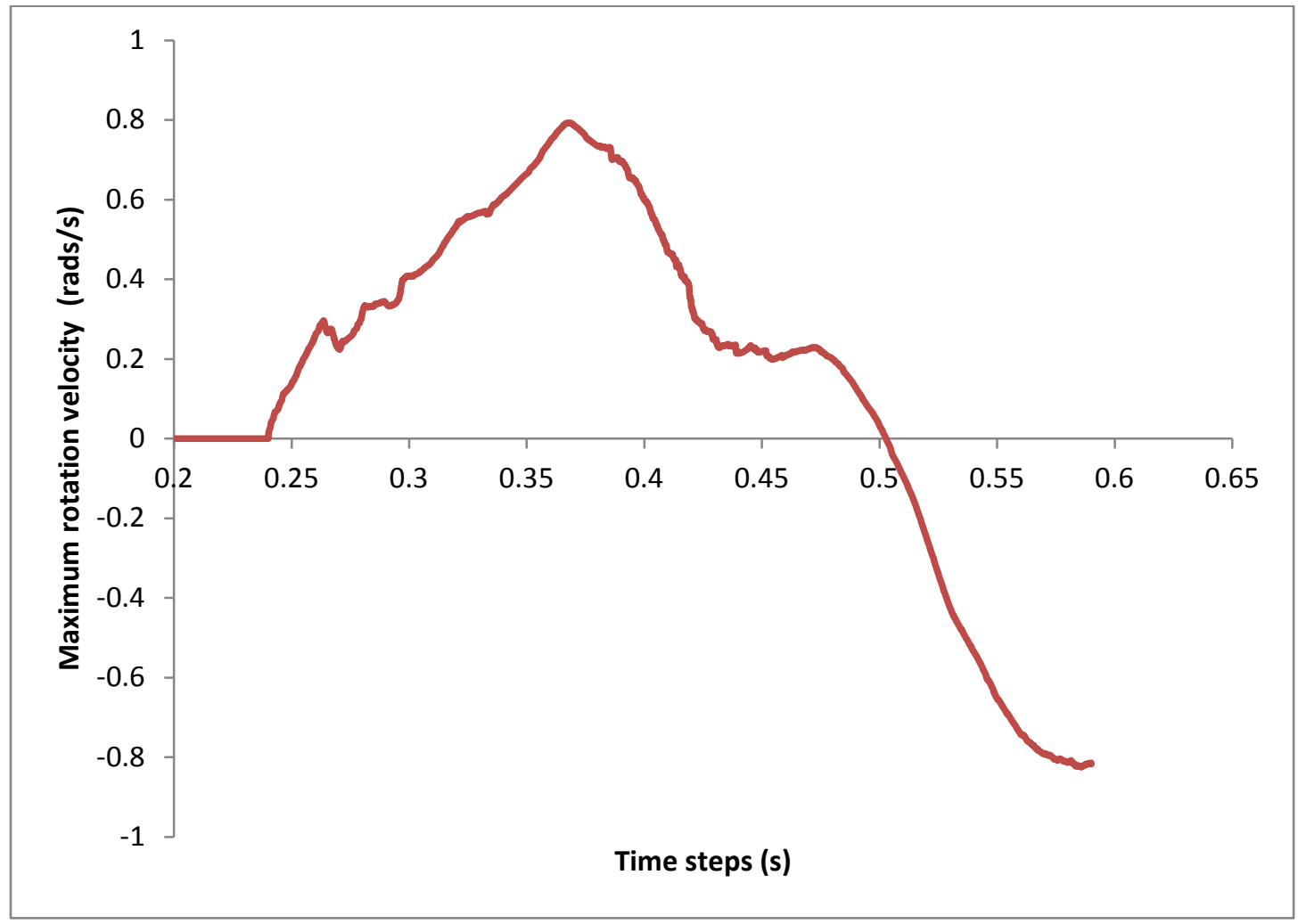

Figure 13: Vessel's rotation velocity before and after impact for current barriers inflated to $7 \mathrm{kPa}$

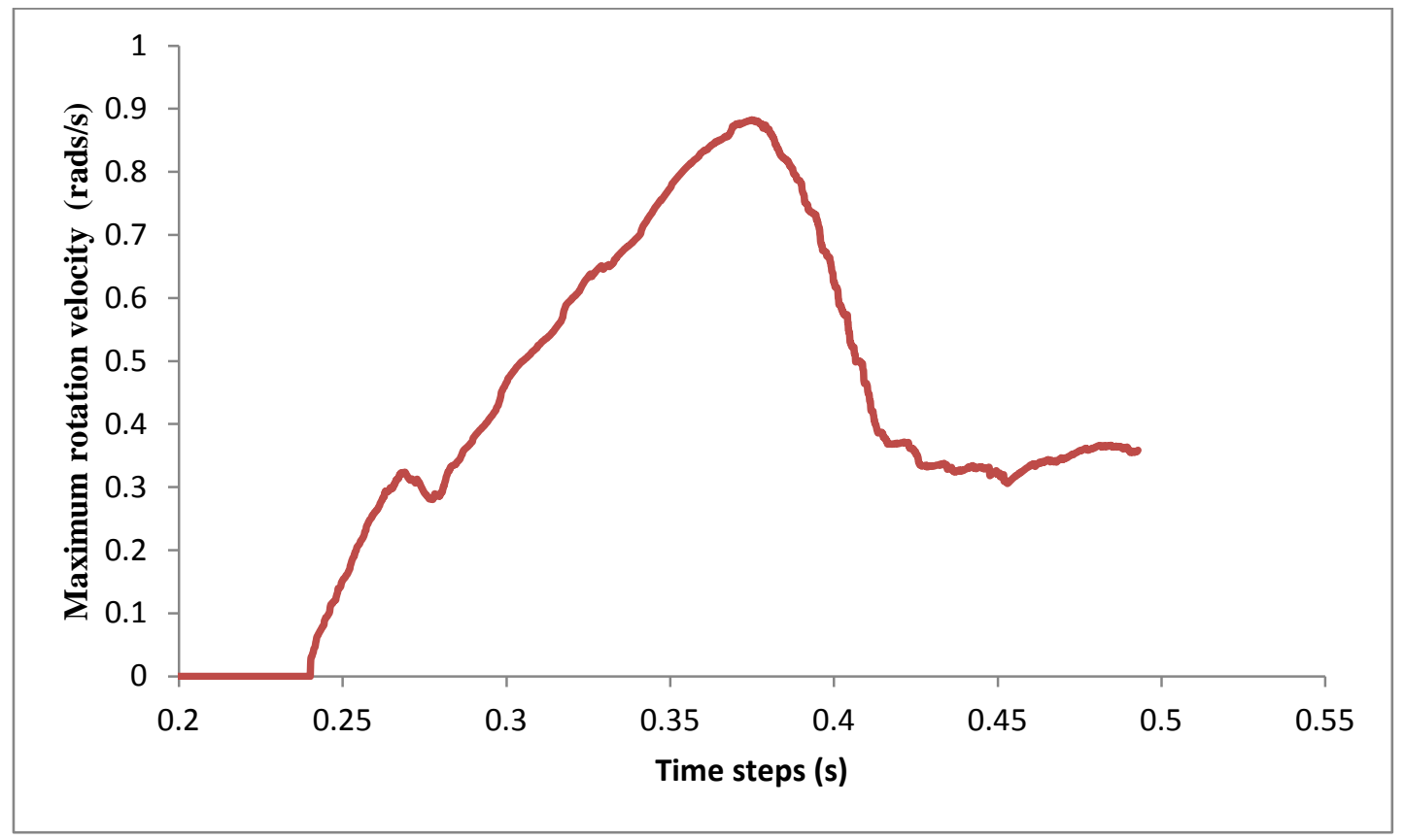

Figure 14: Vessel's rotation velocity before and after impact for modified barriers inflated to $7 \mathrm{kPa}$

- Correspondence author; j.ye2@lancaster.ac.uk 


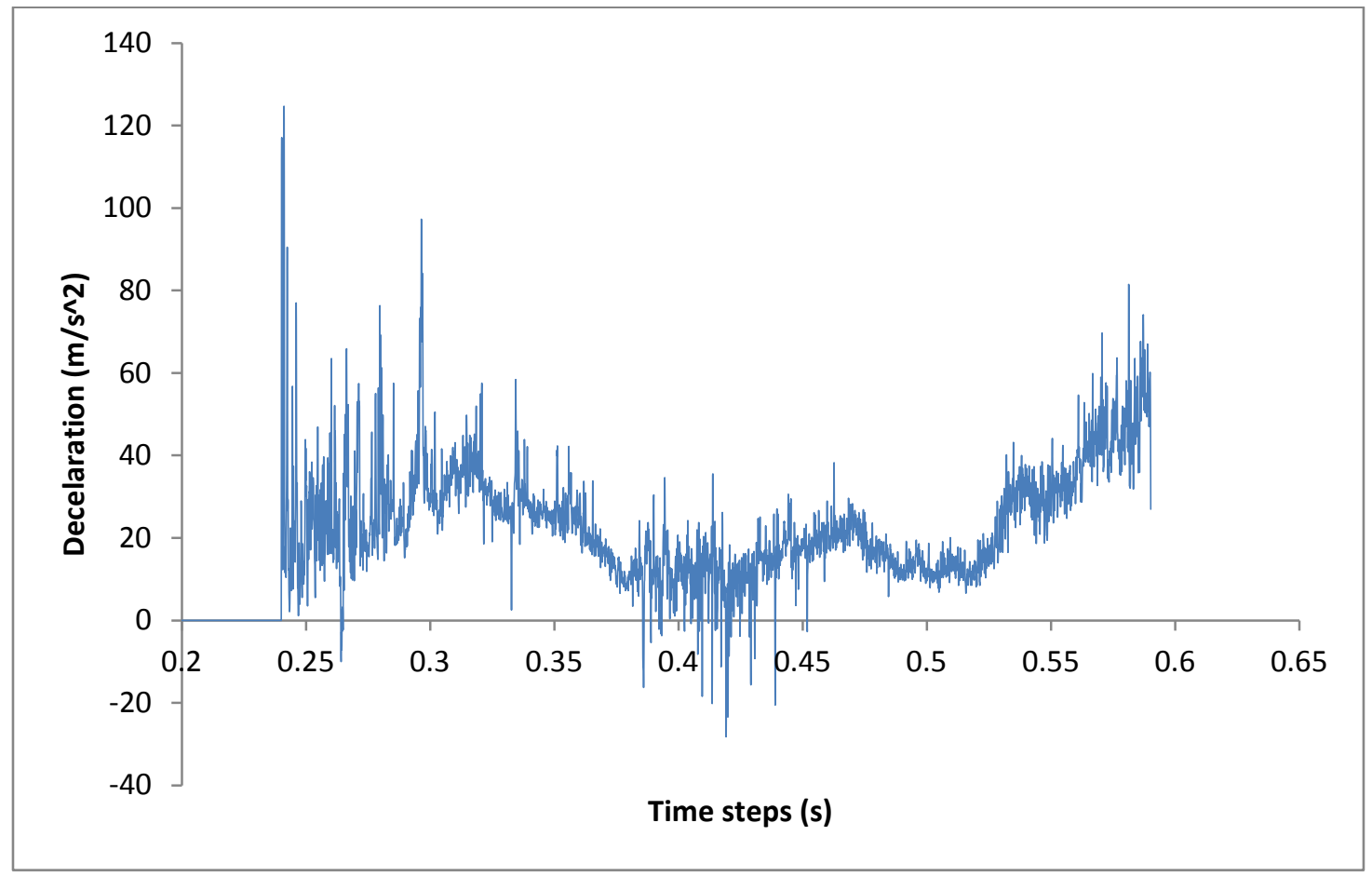

Figure 15: Vessel's deceleration before and after impact of current barriers inflated to $7 \mathrm{kPa}$

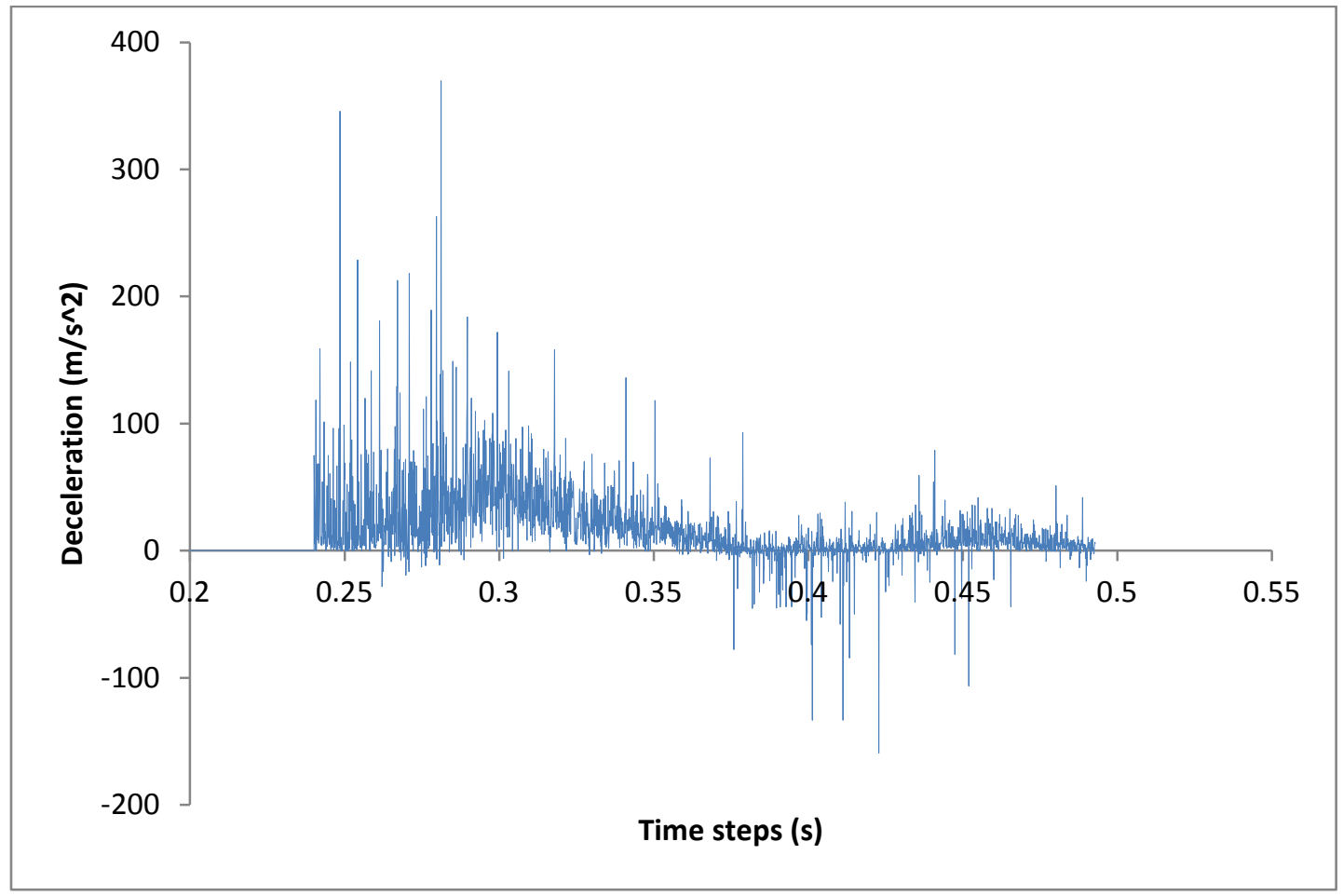

Figure 16: Vessel's deceleration before and after impact of modified barriers inflated to $7 \mathrm{kPa}$.

- Correspondence author; j.ye2@lancaster.ac.uk 
Figures 17 and 18 show velocity loss of the vessel after impact for the current and modified barriers, respectively. The velocity dropped to about $10 \mathrm{~m} / \mathrm{s}$ at $0.3 \mathrm{~s}$ after the impact and remained so in the modified design while the drop in velocity continues after this point in the current design. This is due to the exclusion of reinforcements in the units of the modified design which consequently makes the modified design less dense than the current design, without the additional resistance that would be provided by the internal reinforcements.

To improve on this deficiency while maintaining the plausible gains of the modified design discussed earlier, a hybrid design, encompassing the design provisions in the current and the modified design was further considered.

Results from the hybrid design following impact of the barrier show the gains in the modified and current designs. The drawback however was that high stress concentrations were recorded in areas where the internal reinforcement is in contact with the partition walls as can be seen in Figure 19. The stresses are well beyond the composite material strength and thus indicate potential for the material malfunction. In spite of this local high stress level, the design provision of the hybrid structure is here proposed due to its high potential in meeting the overall design objectives of the structure.

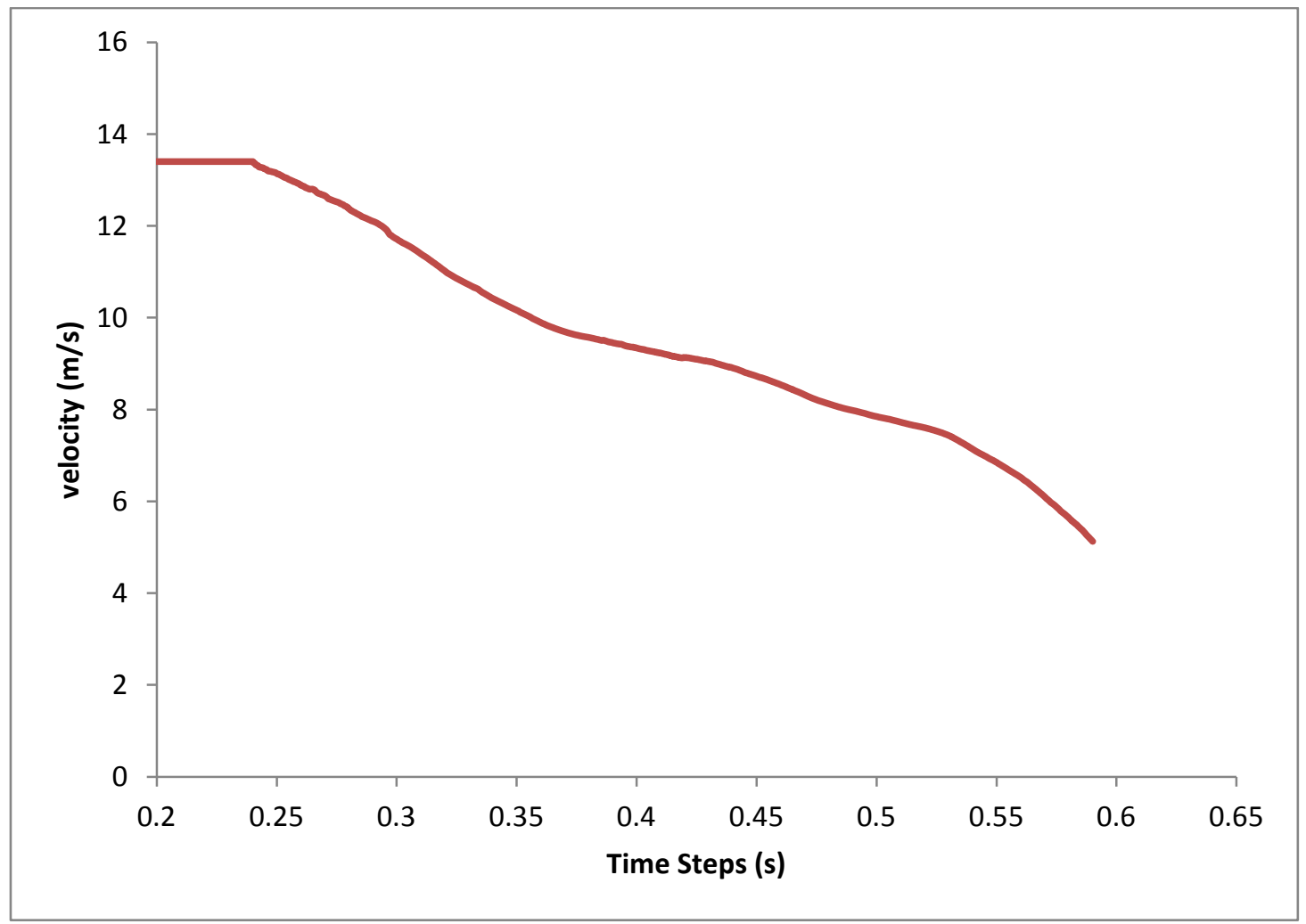

Figure 17: Vessel's impact velocity before and after impact of current barriers inflated to $7 \mathrm{kPa}$

- Correspondence author; j.ye2@lancaster.ac.uk 


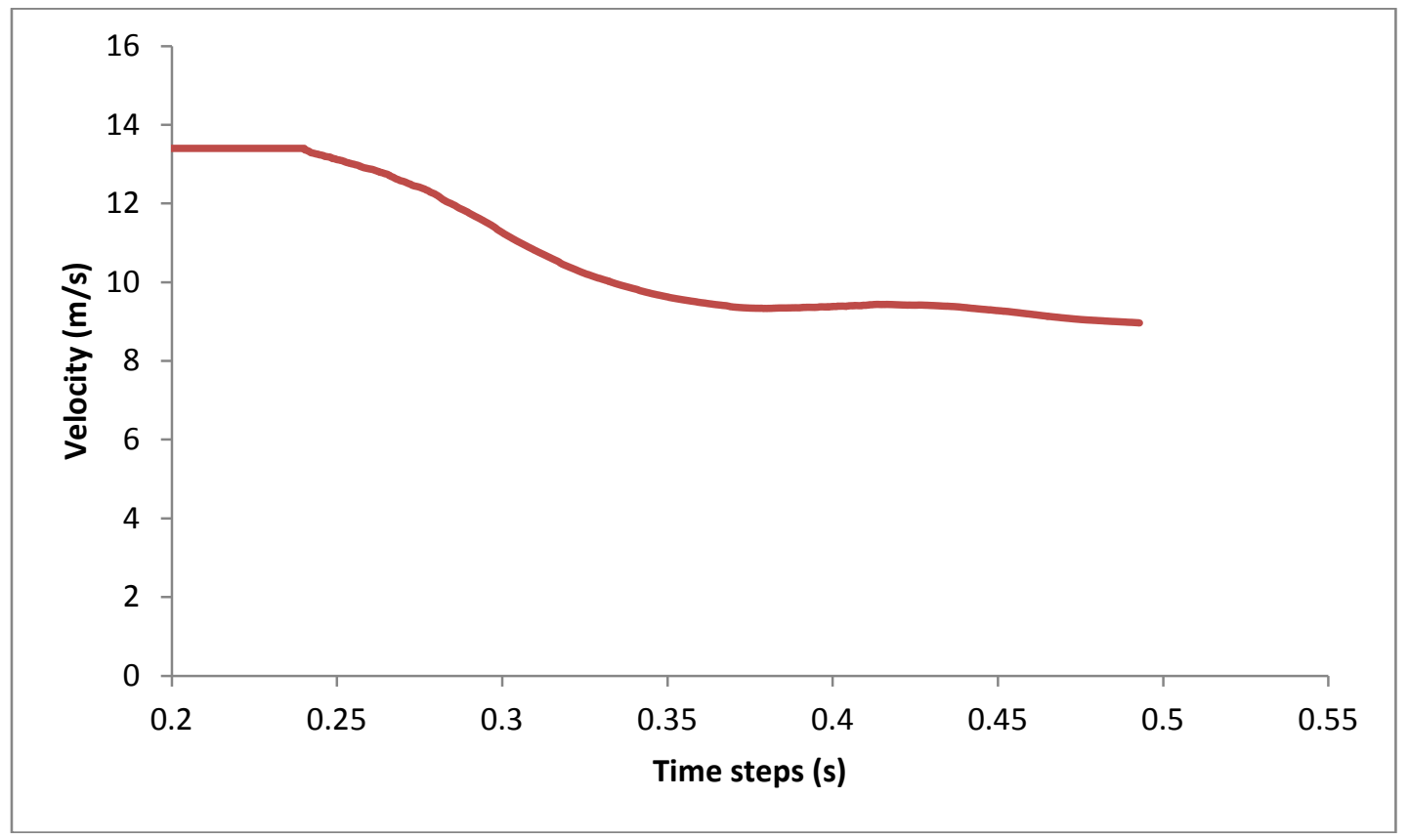

Figure 18: Vessel's impact velocity before and after impact of modified barriers inflated to $7 \mathrm{kPa}$

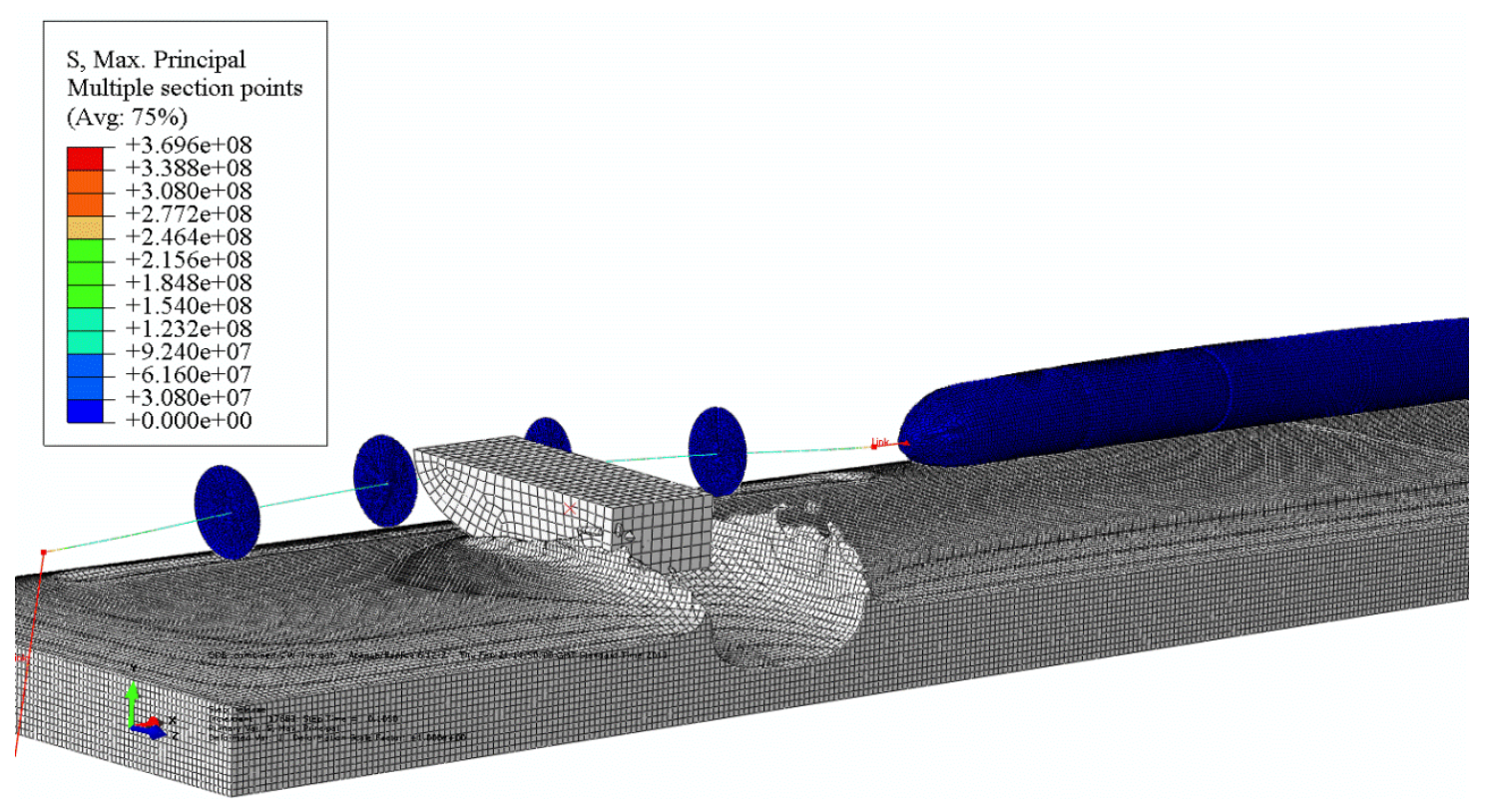

A

Figure 19(A) Maximum principal stress distribution for internal partition walls of modified barriers with $7 \mathrm{kPa}$ initial inflation pressure

- Correspondence author; j.ye2@lancaster.ac.uk 


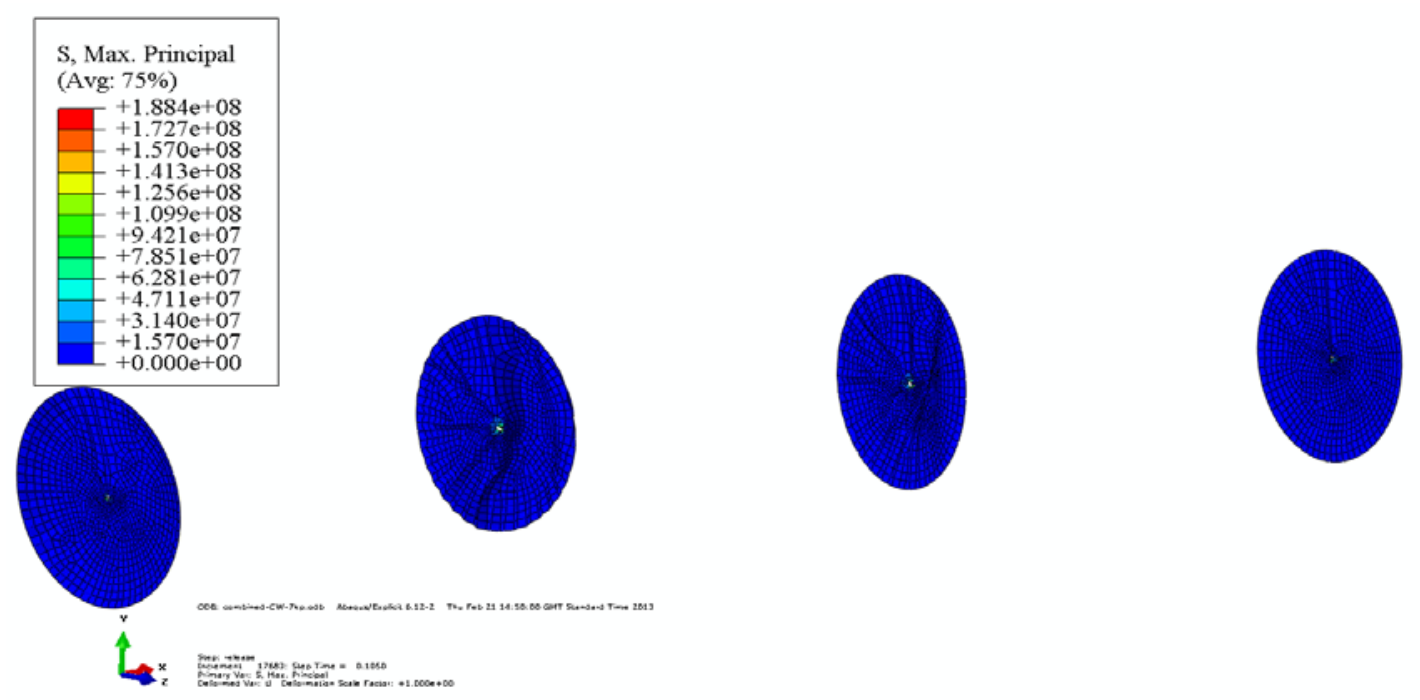

B

Figure 19(B) Maximum principal stress distribution for internal partition walls of modified barriers with $7 \mathrm{kPa}$ initial inflation pressure

\subsection{Conclusion}

In this study an advanced numerical modelling technique for analysis of fluid structure interaction problems is presented.

Using this modelling technique, alternative designs of the inflatable offshore fender barrier structure were considered and performance of the hybrid design described in the body of this work is here proposed.

Numerical simulation of the proposed design as well as the current design show low stress level of the barrier wall after full impact.

The results also suggest that the proposed design has the advantage of dissipating higher kinetic energy, increased vessel instability and deceleration after impact as compared to the current design. These properties are desirable for a barrier structure to effectively prevent boat progress and can be adopted for similar structures

\subsection{Acknowledgement}

The first author is highly indebted to the Management of Petroleum Technology Development Fund for PhD sponsorship at Lancaster University, UK and Dunlop GRG Company for providing some technical details and sample of the composite material used in this study

- Correspondence author; j.ye2@lancaster.ac.uk 


\subsection{References}

[1] Aboshio A., Green S. and Ye J., Experimental Investigation of the Mechanical Properties of Neoprene Coated Nylon Woven Reinforced Composites, Composite Structures, Vol. 120 (2015) 386 to 393

[2] Nixon L.P.E, Synnes D.M.S, B. R, Full scale testing of Dunlop Fender Barriers, in, US Naval Facilities Engineering Services Centre, Port Hueneme, California, 2001.

[3] Sahara reporters, "Militants attacks Exxon Mobil offshore facilities", http://saharareporters.com/news-page/militants-attack-exxonmobil\%E2\%80\%99s-

offshore-facilities -accessed 12/2011

[4] A. Aboshio, S. Green, J. Ye, A New Constitutive Model for Anisotropic Hyperelastic Biased Woven Fibre Reinforced Composite, Plastics, Rubber \& Composites: Macromolecular Engineering, (submitted for review).

[5] Aboshio, Dynamic Study of Inflatable Offshore Barrier Structures under Impact and Environmental Loadings, PhD Thesis, Engineering Department, Lancaster University, United Kingdom, 2014

[6] C. Graczykowski, J. Holnicki-Szulc, Adaptive Inflatable Structures for Impact Absorption, in: III Eccomas Thematic Conference on Smart Structures and Materials, Porland, 2007.

[7] C. Graczykowski, Inflatable Structures for Adaptive Impact Absorption, PhD Thesis, Institute of Fundamental Technological Research Polish Academy of Sciences, 2011.

[8] J. Donea, A. Huerta, J.P. Ponthot, A. Rodr'1guez-Ferran, Arbitrary LagrangianEulerian Methods, Encyclopedia of Computational Mechanics, Edited by Erwin Stein, Ren'e de Borst and Thomas J.R. Hughes. Volume 1: Fundamentals., 1 (2004). [9] E. Kuhl, H. Askes, P. Steinmann, An ALE formulation based on spatial and material settings of continuum mechanics. Part 1: Generic hyperelastic formulation, Computer Methods in Applied Mechanics and Engineering, 193 (2004) 4207-4222.

[10] H. Askes, E. Kuhl, P. Steinmann, An ALE formulation based on spatial and material settings of continuum mechanics. Part 2: Classification and applications, Computer Methods in Applied Mechanics and Engineering, 193 (2004) 4223-4245.

[11] A. Legay, J. Chessa, T. Belytschko, An Eulerian-Lagrangian method for fluidstructure interaction based on level sets, Computer Methods in Applied Mechanics and Engineering, 195 2070-2087.

[12] F. Cirak, R. Radovitzky, A Lagrangian-Eulerian shell-fluid coupling algorithm based on level sets, Comput. Struct., 83 491-498.

[13] N. Aquelet, M. Souli, L. Olovsson, Euler-Lagrange coupling with damping effects:Application to slamming problems, Computer Methods in Applied Mechanics and Engineering, 195 (2006) 110-132.

[14] S. Rugonyi, K.J. Bathe, On Finite Element Analysis of Fluid Flows Fully Coupled with Structural Interactions, CMES, 2 (2001) 195-212.

[15] A. Aboshio, S. Green and J. Ye, "Dynamic Response of Inflatable Offshore Fender Barrier Structures under Impact Loading", in Proceedings of the Fourteenth International Conference on Civil, Structural and Environmental Engineering

- Correspondence author; j.ye2@lancaster.ac.uk 
Computing, B.H.V. Topping and P. Iványi, (Editors), Civil-Comp Press, Stirlingshire, United Kingdom, paper 148, 2013. doi:10.4203/ccp.102.148.

[16] J.N. Reddy, Principles of Continuum Mechanics, Cambridge University Press, New York-USA, 2010.

[17] D. Benson, S. Okazawa, Contact in a multi-material Eulerian finite element formulation, Computer Methods in Applied Mechanics and Engineering, 193 (2004) 4277-4298.

[18] D. Benson, A mixture theory for contact in multi-material Eulerian formulations, Computer Methods in Applied Mechanics and Engineering, 140 (1997) 59-86.

[19] Abaqus Analysis User Manual, Version 6.12.2, Dassault Systems Simulia Corp., Providence, RL.

[20] Dunlop GRG. Boat Barriers. http://www.trelleborg.com/en/DunlopGRG/Products-and-Solutions/AntiTerrorist-Boat-Barriers- Retrieved December 21, 2011

[21] Aboshio A., Green S. and Ye J., Structural Performance Assessment of a Woven-Fabric Reinforced Composite as Applied in Construction of Inflatable Offshore Fender Barrier Structures-International Journal for Structural Stability and Dynamics- Vol. 15, No. 1 (2015) 1450036-1 to 22

[22] A. Aboshio, J. Ye, S. Green, Numerical validation and dynamic study of inflatable offshore barrier structures under impact loading, in: SIMULIA Regional User Meeting, Crewe-United Kingdom, 2013.

- Correspondence author; j.ye2@lancaster.ac.uk 